\title{
Instantes de ocorrência \\ de eventos raros em processos misturadores
}

\author{
Miguel Abadi
}

\author{
TESE APRESENTADA \\ $\mathrm{AO}$ \\ INSTITUTO DE MATEMÁTICA E ESTATÍSTICA \\ $\mathrm{DA}$ \\ UNIVERSIDADE DE SÃO PAULO \\ PARA OBTER O GRAU \\ DE \\ DOUTOR EM ESTATÍSTICA \\ Área de concentração: Probabilidade \\ Orientador: Antonio Galves
}

Durante a realização deste trabalho o autor recebeu suporte financeiro da CAPES

São Paulo, abril de 2001. 



\title{
Instantes de ocorrência de eventos raros em processos misturadores
}

\author{
Este exemplar corresponde à redação \\ final da tese devidamente corrigida \\ e defendida por Miguel Natalio Abadi \\ e aprovada pela comissão julgadora.
}

São Paulo, 27 de abril de 2001.

Banca examinadora:

- Prof. Dr. Antonio Galves, Presidente (IME-USP)

- Prof. Dr. Pablo Augusto Ferrari (IME-USP)

- Prof. Dr. Amine Asselah (UAM)

- Prof. Dr. Nicolai Haydn (USC)

- Prof. Dr. Paul Shields (UT) 



\section{Agradecimentos}

Em primeiro lugar, agradeço a meu orientador, Antonio Galves, por todos os seus esforços, pelas oportunidades de conhecer os mais importantes pesquisadores da área e por ensinarme a ser pesquisador independente.

Agradeço a Adilson, Bernard, Jean-Rene, Nicolai, Pierre, Roberto, Sandro pelas importantes discussões, esclarecimentos e sugerências que ajudaram à realisação desta tese.

Agradeço aos membros do tribunal, Antonio, Pablo, Paul, Nicolai e Amine por ter participado da banca, pelo esforço para entender detenidamente o trabalho, e pelas importantes sugerências e propostas de trabalho indicadas.

Ao personal administrativo da secretaria de estatística e da CPG pela predisposição permanente e o sorriso cotidiano para resolver as inúmeras necessidades planteadas.

A Viviana e Daniel, Jesus, Verónica, Claudia, Washington e Gustavo por ter sido como minha familia no exilio e a meus amigos no IME por fazer do exílio o meu lar.

A Andrea por ter corregido o portuñol, mas por sobre tudo, por ter estado junto a mim estes anos.

$Y$ especialmente a mi familia, porque a la distancia tuvieron siempre palabras de apoyo y estímulo, y guardaron entre ellos las palabras de nostalgia. 


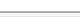




\section{Resumo}

Esta tese trata das distribuições dos processos pontuais formados pelas sucessivas ocorrências de um evento raro em processos estocásticos misturadores.

Este estudo abrange basicamente, três situações bem diferenciadas: o instante de primeira entrada em um evento, o instante de retorno a um evento e o tempo de permanência no evento.

Se considerarmos os instantes das sucessivas ocorrências de um mesmo evento durante a realização de um processo, estes formarão então um processo pontual.

O estudo destas três situações nos permitirá compreender como se distribui esse processo ao longo do tempo para processos estocásticos que perdem memória segundo uma condição geral, que chamamos de processos misturadores. Estes processos abrangem vários tipos de processos estocásticos a tempo discreto bem conhecidos, por exemplo, cadeias de Markov e medidas de Gibbs.

Apresentaremos uma majoração e minoração para o erro na aproximação exponencial para o instante de entrada em um evento raro para processos $\alpha$-misturadores somáveis e processos $\phi$-misturadores quaisquer com alfabeto finito.

Este estudo nos permitirá também fazer uma divisão entre os eventos segundo o comportamento da lei exponencial aproximante.

A lei do instante de volta pode ser aproximada por uma combinação de uma lei degenerada e uma lei exponencial. Por sua vez, a lei do tempo de permanência pode ser aproximada por uma lei geométrica.

Apresentaremos uma estimação do erro em ambos os casos.

Como último resultado obtemos a aproximação poissoniana para os sucessivos instantes de entrada em um evento raro não recorrente para processos $\phi$-misturadores através do método Chen-Stein.

\section{Abstract}

We present upper and lower bound for the error in the exponential approximation of the hitting time law of a rare event in $\alpha$-mixing processes with a summable function $\alpha$ and for general $\phi$-mixing processes with a finite alphabet.

This study will also allow to do a division between events according to the behavior of the 
approximating law.

This thesis deals with the distribution of the point processes of the successive occurrences of a rare event in mixing stochastic processes.

This study consider basically, three very different situations. The hitting time, the return time, and the sojourn time of the event.

The successive occurrence times of the same event during the realization of a process form a point process.

The study of this three situations will allow us to understand how this point process distributes along time in stochastic processes that lose memory according to a general condition called mixing processes.

We also present an estimation of the error in the approximation of the return time to a mixture of a degenerate law and an exponential law and of the sojuorn time by a geometric law.

The final result is the poissonean approximation for the successive occurrences time of a non-recurrent rare event for $\phi$-mixing processes via the Chen-Stein method. 


\section{Índice}

Resumo vii

$\begin{array}{ll}\text { INTRODUÇÃO } & 1\end{array}$

1 ELEMENTOS DE TEORIA ERGÓDICA 7

1.1 Recorrência e ergodicidade . . . . . . . . . . . . . . . . 7

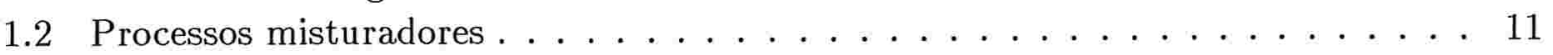

1.2 .1 Definições . . . . . . . . . . . . . . . . . . 11

1.2 .2 Exemplos . . . . . . . . . . . . . . . . . 13

2 RESULTADOS PRÉVIOS 17

2.1 Galves e Schmitt $1997 \ldots \ldots \ldots \ldots \ldots \ldots \ldots$

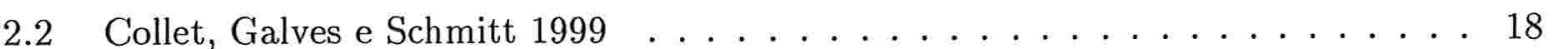

2.3 Hirata, Saussol e Vaienti $1999 \ldots \ldots \ldots \ldots$. . . . . . . . . . . . 18

3 INSTANTE DE ENTRADA

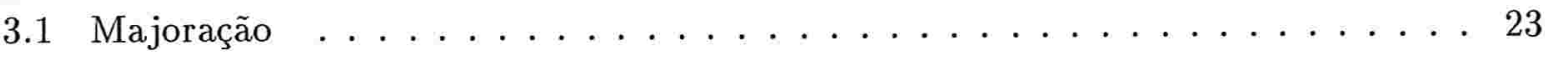

3.1 .1 Resultados . . . . . . . . . . . . . . . . 23

3.1 .2 Estimação da medida de um cilindro . . . . . . . . . . . 25

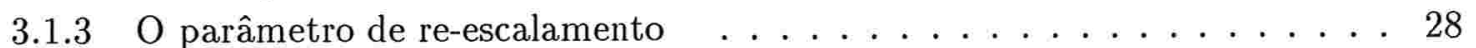

3.1 .4 Propriedade de independência . . . . . . . . . . . . . . . . . . . . . . . . . . . 32

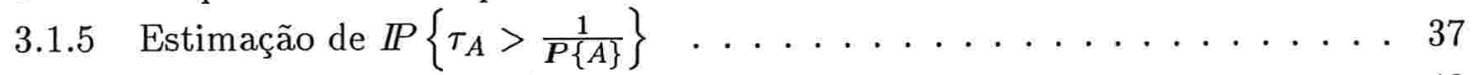

3.1 .6 Majoração em função de $t \ldots \ldots \ldots \ldots \ldots \ldots$. . . . . . . . 40

3.1 .7 Aplicação: Estimação de $\mathbb{E}\left(\tau_{A}\right) \ldots \ldots \ldots \ldots \ldots \ldots$. . . . . . . . . . . . . . . . . .

3.2 Minoração uniforme . . . . . . . . . . . . . . . . . 45

3.3 Comportamento do parâmetro de re-escalamento . . . . . . . . . . . . 47

3.3.1 Estimação do parâmetro de re-escalamento . . . . . . . . . . . . . 47

3.3 .2 Limite quase-certo . . . . . . . . . . . . . . . . . . . 50

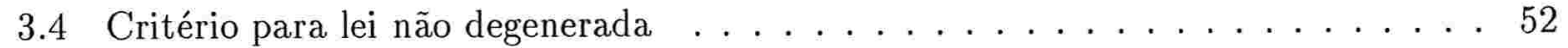

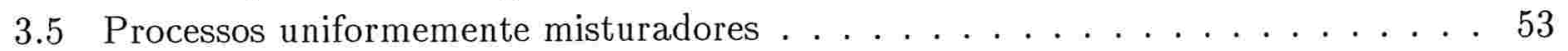


4 INSTANTE DE VOLTA E TEMPO DE PERMANÊNCIA 57

4.1 Motivação . . . . . . . . . . . . . . . . . . . 57

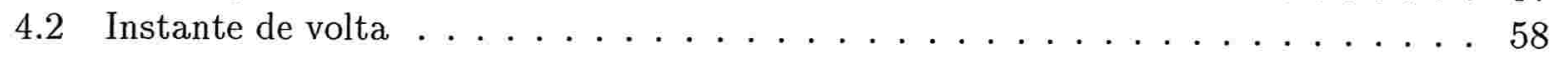

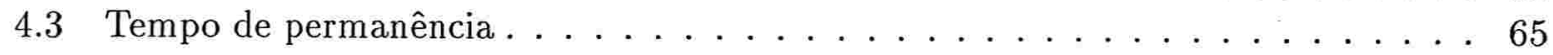

5 PROCESSO DE POISSON 69

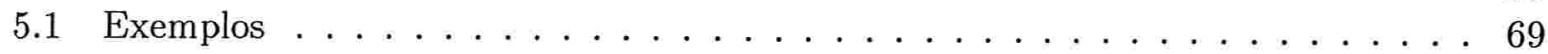

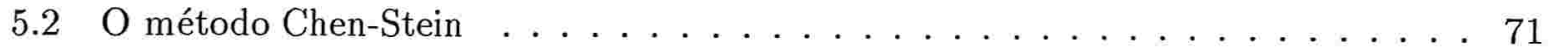

5.3 Convergência a um processo de Poisson para processos misturadores . . . . . 73

$\begin{array}{ll}\text { Referências Bibliográficas } & 81\end{array}$ 


\section{INTRODUÇÃO}

O primeiro instante de ocorrência de um evento raro em um processo estocástico misturador possui uma distribuição que pode ser bem aproximada pela lei exponencial .

Nesta tese, apresentaremos resultados que mostram expressões para o erro cometido nesta aproximação.

O instante de volta e o processo das sucessivas entradas no evento raro são aproximados por: uma mistura duma lei exponencial e uma medida de Dirac, no primeiro caso, e por uma lei geométrica no segundo. Já, o processo das sucessivas entradas pode ser aproximado por uma lei de Poisson.

Apresentaremos também resultados que mostram expressões para esses erros nestas aproximações.

O pioneiro nesta área é Döeblin (1940), que estudou a aproximação de Poisson para a transformada de Gauss. No contexto de cadeias de Markov, a convergência do instante de ocorrência de um evento raro à lei exponencial foi primeiro estudada por Bellmann e Harris (1951) e Harris (1953).

Então, depois de um longo período no qual, aparentemente, nada apareceu na área, vários artigos e livros estudando o problema para diferentes tipos de processos markovianos foram publicados, começando com Chen (1975), Keilson (1979), Aldous (1982), Korolyuk e Sil'vestrov (1984), Cassandro, Galves, Olivieri e Vares (1984), Kipnis e Newman (1985), Cogburn (1985), Lebowitz e Schonmann (1987), Olivieri e Scoppola (1995) e (1996) entre outros.

No contexto de sistemas dinâmicos a questão foi considerada por Galves e Schmitt (1990), Pitskel (1991), Collet, Galves e Schmitt (1992), Collet e Galves (1993), Hirata (1993) e Collet e Galves (1995).

Parte desses trabalhos foi motivada pela modelagem dos fenômenos físicos de metastabilidade e intermitência.

Com exceção de Chen (1975) e Aldous (1982), todos os outros estão interessados em resultados qualitativos e não apresentam expressões para o erro na aproximação exponencial. 
Já na década do noventa, vários artigos apresentam majorações para o erro na aproximação exponencial. Aldous e Brown $(1992,1993)$ estudam o caso de cadeias de Markov reversíveis finitas.

Resultados para diferentes tipos de sistemas markovianos interatuantes aparecem em Ferrari, Galves e Landim (1994), Ferrari, Galves e Liggett (1995), Asselah e Dai Pra (1997a e b), Simonis (1998).

O assunto é considerado no contexto de diferentes tipos de processos misturadores em trabalhos recentes por diferentes autores.

Haydn (1999) trabalha com mapas racionais sobre a esfera de Riemann. Paccaut (1999) considera o caso de mapas do intervalo com peso.

Galves e Schmitt (1997) consideram sistemas dinâmicos $\phi$-misturadores com respeito de uma função somável $\phi$. Eles introduzem um fator de correção na lei aproximante. A técnica funciona para toda classe de cilindros, mas só no caso de processos $\phi$-misturadores com função somável $\phi$.

Collet, Galves e Schmitt (1999) consideram estados de Gibbs unidimensionais, com potencial Hölder contínuo. Eles mostram que se o evento é não recorrente, então, o parâmetro na lei é um, e que essa é a situação típica para processos exponencialmente $\phi$-misturadores.

Hirata, Saussol e Vaienti (1999) provam a aproximação de Poisson para os sucessivos instantes de entrada em um evento raro. Sua prova vale em um marco muito geral, embora o resultado seja interessante para cilindros que não recorram numa vizinhança do cilindro considerado.

Faremos uma breve apresentação de tais resultados no Capítulo 2. Para uma breve referência na área sobre os trabalhos publicados antes de 1997, referimos o leitor a Galves e Schmitt (1997). Para um repasso detalhado dos trabalhos que apresentam majorações para a aproximação exponencial em um marco geral, referimos o leitor a Abadi e Galves (2000).

Por outro lado, é interessante observar que, no caso de cadeias de Markov nulas recorrentes a aproximação exponencial poderia não valer. Este assunto foi recentemente trabalhado por Bressaud e Zweimüller (2000). Eles consideram uma família de transformações afins por partes do intervalo com um ponto fixo indiferente. Eles mostram que o instante da primeira visita a um pequeno intervalo ao redor de um ponto fixo fortemente repelente, não converge para uma lei exponencial. Mais precisamente, apresentam condições que garantem que a lei limite é o produto de uma lei exponencial a alguma potência e uma lei estável. Pelo que sabemos, esse é o primeiro resultado desse tipo que foi provado para sistemas de alta complexidade.

Uma aplicação da aproximação exponencial é o estudo das flutuações dos instantes de volta para uma sequência longa de símbolos. Isto foi motivado pelo estudo das propriedades assintóticas dos algoritmos de compressão como o de Lempel-Ziv. Isto foi feito em Collet, Galves e Schmitt (1999) para o caso de fontes gibbsianas e em Collet (2000), no caso de aplicações não uniformemente hiperbólicos. 
Nesta tese apresentaremos majorações e minorações para o erro na aproximação da lei exponencial para a lei do primeiro instante de ocorrência de uma sequência longa de símbolos num processo estocástico com alfabeto finito.

O resultado vale para processos $\alpha$-misturadores com função $\alpha$ somável, para processos $\phi$ misturadores com função decrescente qualquer e para eventos não recorrentes em processos $\gamma$ ou uniformemente misturadores em que a função $\gamma$ decresce, pelo menos, com a velocidade com que decrescem os cilindros.

Provaremos que para todo conjunto mensurável cilíndrico $A$, a lei do seu instante de entrada, adequadamente re-escalada, pode ser uniformemente aproximada por uma lei exponencial de média um. O erro na aproximação desta lei é minorado por $C_{1} \mathbb{P}\{A\}$. É majorado por uma função que depende de $\mathbb{I P}\{A\}$ e da função de perda de correlação do processo onde $\mathbb{P}\{A\}$ é a medida do cilindro.

Mais ainda, mostraremos que o fator de escala pode ser limitado por duas constantes estritamente positivas $\Lambda_{1}$ e $\Lambda_{2}$, independentes de $A, n$.

O Lema de Kac (Kac, 1947) diz que o valor correto para a re-escala do instante de volta é $I P\{A\}$. Isto sugere usá-lo como fator de re-escala para o instante de entrada, o que em nossa notação significa $\xi_{A}=1$. Mostraremos que esse não é sempre o caso para instantes de entrada. Estudaremos como $\xi_{A}$ depende de $A$. Nesse sentido, o uso do valor $\xi_{A}$ dá uma boa aproximação da lei exponencial mas pode ser difícil de calculá-lo explicitamente. Para enfrentar essa dificuldade, apresentaremos uma estimação calculável do parâmetro em termos dos instantes curtos de recorrência do cilindro.

Mais ainda, no caso dos processos $\alpha$-misturadores e $\phi$-misturadores apresentaremos uma majoração não uniforme e sim em função de $t$.

Isso será feito mediante um refinamento da técnica usada por Galves e Schmitt. Ela mostra como a velocidade de convergência depende da escolha adequada do parâmetro no re-escalamento da lei. Ela também mostra não uma majoração uniforme do erro mas uma majoração em função do tempo $t$. Esta nova visão é fundamental para posteriores resultados. Um deles é o controle do decaimento da cauda da lei do instante de entrada. Uma outra importante consequência do erro na aproximação exponencial em função de $t$ é a estimação da esperança do tempo de entrada no evento raro.

Esta nova técnica é válida para todo tipo de cilindros e para uma família mais ampla de processos misturadores.

Doukhan (1995) garante a existência de processos satisfazendo estritamente estas condições generalizadas o que garante o sentido de tais extensões aos resultados anteriores.

Um dos objetivos desta generalização é responder a uma das perguntas apontadas no artigo de Hirata, Saussol e Vaienti: Qual é a maior classe de processos para os quais vale a lei exponencial? Outra motivação é a seguinte: se enxergarmos qual é a condição mínima suficiente para 
valer a aproximação exponencial, ela nos mostrará qual é a recíproca que se pode esperar destes teoremas, isto é, se vale a aproximação exponencial, que tipo de mistura pode ser garantida no processo.

Um corolário apresentará condições, sobre o processo e sobre o cilindro, para obter o parâmetro $\xi_{A}$ igual a um; assim como que a medida daqueles cilindros para os quais $\xi_{A}$ não é um, decai exponencialmente. Este comportamento já foi indicado em Hirata, Saussol, Vaienti (1999), Collet, Galves, Schmitt (1999) e Saussol (1999). Apresentaremos um exemplo no qual não somente o parâmetro $\xi_{A}$ é distinto de 1, mas também, tão pequeno quanto se queira (de acordo com a medida de equilíbrio do processo).

Uma das condições que garante o controle do parâmetro $\xi_{A}$ é dada pelo controle do momento de segunda ordem da função de contagem de ocorrências do evento $A$. Uma pergunta que permanecia aberta é se essa condição era necessária. Nós provaremos que o controle do parâmetro equivale ao controle do que poderíamos chamar de momento de ordem logarítmico mais um.

Um dos interesses é estimar a ordem exata de convergência para a lei exponencial. Com esse objetivo é que apresentaremos uma minoração uniforme do erro na aproximação exponencial.

O último resultado do capítulo é a convergência para a lei exponencial para processos uniformemente misturadores com decaimento de correlação da ordem do decaimento da medida dos cilindros não recorrentes.

O interesse por descrever o processo pontual das ocorrências de um evento raro nos leva a considerar o problema não já do instante de entrada, mas do instante de volta e posterior tempo de permanência em um evento raro. Através do resultado para instantes de entrada, veremos que a lei do instante de volta pode ser aproximada por uma mistura de uma lei degenerada, isto é, uma medida de Dirac, concentrada em um ponto que depende das propriedades de recorrência do evento e uma lei exponencial. Portanto, as leis de entrada e retorno coincidirão somente quando o evento for do tipo não recorrente e, neste caso, a medida de Dirac estará ausente.

O fato de termos uma majoração para o erro na aproximação do instante de entrada nos permitirá obter um erro na aproximação do instante de volta.

Num quadro geral, esse tipo de comportamento já foi indicado por Pitskel (1991). Nesse trabalho, é provado o limite assintótico quase-certo para uma lei de Poisson para cadeias de Markov irredutíveis e aperiódicas com espaço de estados finito. Mostra-se que a condição quasecerta é essencial, pois pontos fixos contradizem o resultado.

Hirata (1993) mostra o limite assintótico quase-certo para uma lei de Poisson para difeomorfismos Axioma A. Entanto para pontos periódicos prova, sem velocidade de convergência, a convergência do primeiro tempo de volta para uma combinação de uma lei exponencial e uma medida de Dirac. 
Já Hirata, Saussol e Vaienti (1999) mostram que a lei de entrada e lei de retorno convergem para uma mesma lei se e somente se a lei de retorno converge para uma lei exponencial. No corolário 4.5, nos mostraremos que a condição para que a lei de retorno convirja para uma lei puramente exponencial equivale a considerar eventos não recorrente.

O tempo de volta nos leva a considerar um segundo problema: o da lei do tempo de permanência no evento. Veremos que, sobre certa condição de regularidade da medida, esta lei pode ser bem aproximada por uma lei geométrica.

Isto será considerado no Capítulo 4.

No Capítulo 5, estudaremos a convergência do processo pontual das sucessivas ocorrências de um evento raro para uma lei de Poisson para eventos não recorrentes, utilizando o método Chen-Stein.

Esta é uma generalização da convergência da distribuição binomial de parâmetros $n$ e $p$ para a distribuição de Poisson de parâmetro $\lambda$ quando $n$ diverge e $p$ é pequeno satisfazendo $\lambda=n p$.

Stein (1972) apresentou uma nova técnica para mostrar a convergência em distribuição a uma normal para soma de variáveis aleatórias dependentes. O objetivo é a generalização do Teorema de Limite Central para casos não independentes.

Em 1975, Chen usou a técnica de Stein para estudar a aproximação binomial à distribuição de Poisson no caso de variáveis dependentes.

Embora o método Chen-Stein prove uma majoração do erro na aproximação da distribuição dos instantes de ocorrências de um evento raro à distribuição de Poisson, a majoração não é totalmente transparente e não garante em que casos o erro converge para zero.

Aplicamos então, o método Chen-Stein aos processos $\phi$-misturadores considerando eventos não recorrentes e provamos assim, a convergência para uma lei de Poisson. Com um exemplo mostramos que o método não converge se se considera um evento recorrente. 


\section{Capítulo 1}

\section{ELEMENTOS DE TEORIA ERGÓDICA}

\subsection{Recorrência e ergodicidade}

Estabeleceremos primeiramente algumas definições e conceitos básicos que nos serão úteis para apresentar nosso marco de trabalho e resultados.

Seja $\mathcal{E}$ um conjunto finito que chamaremos de alfabeto. Denotamos $\Omega=\mathcal{E}^{\mathbb{Z}}$. Para cada $n \in \mathbb{Z}$, definimos a variável aleatória $X_{n}: \Omega \rightarrow \Re$ como a $n$-ésima coordenada de um $\omega \in \Omega$, isto é, se $\omega=\left\{\omega_{n}\right\}_{n \in \mathbb{Z}}$, então $X_{n}(\omega)=\omega_{n}$ para todo $n \in \mathbb{Z}$.

Para cada subconjunto $A$ de $\Omega$ da forma

$$
A=\left\{X_{0}=a_{0}, \ldots, X_{n-1}=a_{n-1}\right\}
$$

com $a_{i} \in \mathcal{E}, i=1, \ldots, n$, diremos que $A$ é um cilindro de ordem $n$ ou que $A$ é um $n$-cilindro.

Denotaremos com $\mathcal{C}_{n}$ o conjunto de todos os $n$-cilindros. Denotaremos também com $\mathcal{F}$ a $\sigma$ álgebra definida sobre $\Omega$ e gerada pelos cilindros. Mais ainda, denotaremos com $\mathcal{F}_{I}$ a $\sigma$-álgebra gerada pelos cilindros com coordenadas em $I, I \subseteq \mathbb{Z}$. Seja $\mathbb{P}$ uma medida de probabilidade definida sobre a $\sigma$-álgebra $\mathcal{F}$. Desta forma $(\Omega, \mathcal{F}, \mathbb{P})$ é um espaço de medida e $\left\{X_{m}\right\}_{m \in \mathbb{Z}}$ é um processo estocástico.

Denotaremos com $T: \Omega \rightarrow \Omega$ o operador de translação à esquerda em uma coordenada, isto é, $(T(\omega))_{n}=\omega_{n+1}$, para todo $\omega \in \Omega$ e para todo $n \in \mathbb{Z}$. 
Definição 1.1 Seja IP uma medida de probabilidade sobre $\mathcal{F}$, tal que

$$
\mathbb{P}\{A\}=\mathbb{P}\left\{T^{-1}(A)\right\}
$$

para todo $A \in \mathcal{F}$. IP será chamada uma medida estacionária ou equivalentemente diremos que $\left\{X_{m}\right\}_{m \in \mathbb{Z}}$ é um processo estacionário.

Definição 1.2 Seja $A \in \mathcal{F}$. Diremos que $A$ é invariante (para o operador $T$ ) se e somente se

$$
T^{-1}(A)=A
$$

Esta definição permite-nos introduzir o conceito de ergodicidade.

Definição 1.3 O processo $\left\{X_{m}\right\}_{m \in \mathbb{Z}}$ será dito ergódico se os únicos subconjuntos mensuráveis invariantes de $\Omega$, possuem medida 0 ou 1 .

Ergodicidade é então, uma noção que nos permite falar da indecomposição do processo. Isto é, não existem conjuntos mensuráveis $A \subseteq \Omega$, que sejam invariantes pelo operador $T$ sem que possuam medida zero ou um, ou dito informalmente, sem que sejam o vazio ou $\Omega, I P$-quasecertamente.

Definição 1.4 Dado $A \in \mathcal{C}_{n}$, definimos o tempo de entrada em $A$ como sendo a variável aleatória $\tau_{A}: \Omega \rightarrow \mathbb{N} \cup\{\infty\}$ dada por

$$
\tau_{A}(\omega)=\inf \left\{k \geq 1: T^{k}(\omega) \in A\right\}
$$

A ergodicidade de um processo nos garante que podemos esperar a ocorrência de um evento de medida positiva em quase toda realização do processo. 
Proposição 1.5 Seja $\left\{X_{m}\right\}_{m \in \mathbb{Z}}$ um processo ergódico. Seja A tal que $\mathbb{P}\{A\}>0$. Então

$$
\mathbb{I P}\left\{\omega \in \Omega: \tau_{A}(\omega)<\infty\right\}=1
$$

Estaremos interessados não somente em falar de tempos de entrada, mas também, em tempos de volta.

Definição 1.6 Para um evento $A$ de medida estritamente positiva, definimos a medida condicional com respeito ao evento $A$ como sendo

$$
\mathbb{P}_{A}\{W\}=\mathbb{P}\{A \cap W\} / \mathbb{P}\{A\}
$$

para todo $W \subseteq \mathcal{F}$.

Agora, se considerarmos a $\sigma$-álgebra $\mathcal{F}_{A}$ dos mensuráveis $B \subseteq \mathcal{F}$ restringidos a $A$, isto é, $\mathcal{F}_{A}=\{B \cap A: B \subseteq \mathcal{F}\}$, temos que $\left(A, \mathcal{F}_{A}, \mathbb{P}_{A}\right)$ é um espaço de medida no qual podemos considerar o operador $\left.T\right|_{A}: A \rightarrow A$.

Definição 1.7 Dado $A \in \mathcal{C}_{n}$, definimos o tempo de volta $A O$ evento $A$ como sendo a variável aleatória $\left.\tau_{A}\right|_{A}: A \rightarrow \mathbb{I N} \cup\{\infty\}$ dada por

$$
\left.\tau_{A}(\omega)\right|_{A}=\inf \left\{k \geq 1: T^{k}(\omega) \in A\right\}
$$

que por um abuso de notação, quando não houver ambiguidade, escreveremos simplesmente $\tau_{A}$.

O fundamental Principio de Recorrência de Poincaré garante-nos que em quase toda realização do processo em que acontece $A$, voltará a acontecer $A$, mais ainda, ele acontecerá infinitas vezes.

Teorema 1.8 (Poincaré) Seja $\left\{X_{m}\right\}_{m \in \mathbb{Z}}$ um processo estacionário e seja $A$ um conjunto mensurável de $\Omega$. Então, para quase todo $\omega \in A, T_{h}^{k}(\omega) \in A$ para algakm $k>0$. 
Vamos agora apresentar o célebre Lema de Kac.

Lema 1.9 (Kac) Seja $\left\{X_{m}\right\}_{m \in \mathbb{Z}}$ um processo ergódico e A um conjunto mensurável tal que $\mathbb{I P}\{A\}>0$. Então,

$$
\frac{1}{\mathbb{P}\{A\}}=\mathbb{E}_{A}\left(\tau_{A}\right)
$$

onde $\mathbb{E}_{A}$ denota a esperança condicional com respeito a $A$.

Um conceito importante que fala sobre a medida da complexidade de um processo é o conceito de entropia.

Definição 1.10 A entropia de um processo em relação ao alfabeto $\mathcal{E}$ é o número

$$
h(\mathcal{E})=-\sum_{a \in \mathcal{E}} \mathbb{P}\{a\} \log \mathbb{I P}\{a\}
$$

onde adotamos a convenção $0 \log 0=0$ e onde $\log$ é o logaritmo natural.

Definição 1.11 A entropia de um processo com relação ao operador $T$ é o número

$$
h(T)=\sup _{n \in N} h\left(\vee_{k=1}^{n} T^{-k} \mathcal{E}\right)
$$

Teorema 1.12 (Shannon-Mc Millan-Breinman) Suponhamos que o processo $\left\{X_{m}\right\}_{m \in \mathbb{Z}}$ seja ergódico. Para quase todo $\omega \in \Omega$ (respeito da medida $\mathbb{P}$ )

$$
\lim _{n \rightarrow \infty} \frac{1}{n} \log \mathbb{P}\left\{A_{n}(\omega)\right\}=-h(T) .
$$

onde $A_{n}(\omega)$ é o n-cilindro de coordenadas $\omega_{1}, \ldots, \omega_{n}$. 
Este resultado nos diz que um cilindro de tamanho $n$ terá medida aproximadamente $e^{-h n}$. Em consequência, temos que o número de cilindros de comprimento $n$ será aproximadamente $e^{h n}$. Deste ponto de vista, dizemos que a entropia é uma medida da complexidade do processo.

Usaremos a clássica notação probabilista para eventos definidos através de variáveis aleatórias. Escreveremos $\left\{\tau_{A}=m\right\}$ em lugar de $\left\{\omega \in \Omega: \tau_{A}(\omega)=m\right\}$, e $T^{-k}(A)$ ou $\left\{T^{k} \in A\right\}$ em lugar de $\left\{\omega \in \Omega: T^{k}(\omega) \in A\right\}$. Como é usual, a média da variável aleatória $X$ será chamada de esperança e denotada por $\mathbb{E}(X)$.

As provas dos resultados acima mencionados, com exceção do Teorema 1.12, podem ser achadas em Cornfeld, Fomin e Sinai (1982). A prova do Teorema de Shannon-Mc MillanBreinman pode ser encontrada em Billingsley (1965).

\subsection{Processos misturadores}

\subsubsection{Definições}

Para estudar a distribuição do instante de entrada e instante de volta é preciso definir que tipo de perda de memória possui o processo.

Definição 1.13 Diremos que $\left\{X_{m}\right\}_{m \in \mathbb{Z}}$ é

1. $\gamma$ ou uniformemente misturador se, para todos os inteiros $n \geq 1$ el $l \geq 0$, a seguinte desigualdade é satisfeita

$$
\sup _{B \in \mathcal{F}_{\{0, ., n\}}, C \in \mathcal{F}_{\{n \geq 0\}}}\left|\mathbb{P}\left\{B \cap T^{-(n+l+1)} C\right\}-\mathbb{P}\{B\} \mathbb{P}\{C\}\right|=\gamma(l)
$$

2. $\alpha$-misturador se

$$
\sup _{B \in \mathcal{F}_{\{0, ., n\}}, C \in \mathcal{F}_{\{n \geq 0\}}} \frac{\left|\mathbb{P}\left\{B \cap T^{-(n+l+1)} C\right\}-\mathbb{P}\{B\} \mathbb{P}\{C\}\right|}{\mathbb{P}\{B\}}=\alpha(l),
$$

\section{$e$}


3. $\phi$-misturador se

$$
\sup _{B \in \mathcal{F}_{\{0,, n\}}, C \in \mathcal{F}_{\{n \geq 0\}}} \frac{\left|\mathbb{P}\left\{B \cap T^{-(n+l+1)} C\right\}-\mathbb{P}\{B\} \mathbb{P}\{C\}\right|}{\mathbb{P}\{B\} \mathbb{P}\{C\}}=\phi(l)
$$

onde nas expressões acima, o supremo é tomado sobre os conjuntos $B$ e $C$, tal que $\mathbb{I P}\{B\}>0$ no segundo caso e tal que $\mathbb{I P}\{B\} \mathbb{P}\{C\}>0$ no terceiro caso, e onde $\gamma=(\gamma(l))_{l \geq 0}, \alpha=(\alpha(l))_{l \geq 0}$ e $\phi=(\phi(l))_{l \geq 0}$, são sequências decrescentes de números reais positivos tendendo a zero. forme.

Claramente, $\phi$-misturador implica $\alpha$-misturador que, por sua vez, implica misturador uni-

Observação Na literatura, as diferentes condições de mistura têm recebido nomes diferentes. Por exemplo, Doukhan (1995) chama de $\alpha$-misturador (respectivamente $\phi, \psi$ ), ao que nós chamamos $\gamma$-misturador (respectivamente $\alpha, \phi$ ). Também, Shields (1996) usa $\psi$-misturador para denotar o que nós chamamos de $\phi$-misturador. Philipp e Stout (1975) chamam de $\phi$ misturador ao que nós chamamos $\alpha$-misturador e ao que nós chamamos $\gamma$-misturador, eles chamam de $\beta$-misturador ou misturador forte.

Nossa terminologia é a mesma adotada por Galves e Schmitt (1997); Collet, Galves e Schmitt (1999) e Hirata, Saussol e Vaienti (1999).

É importante notar que qualquer processo misturador é ergódico.

Lema 1.14 Seja $\left\{X_{m}\right\}_{m \in \mathbb{Z}}$ um processo uniformemente misturador com função decrescente $\gamma$ qualquer. Então, o processo é ergódico.

Prova. Suponhamos que $A$ é invariante. Devemos assumir que o processo é uniformemente misturador sem assumir nenhuma hipótese a respeito do decaimento da função $\gamma$, ou seja, temos simplesmente que

$$
\lim _{l \rightarrow \infty} \mathbb{P}\left\{B \cap T^{-(n+l+1)} C\right\}=\mathbb{P}\{B\} \mathbb{P}\{C\}
$$

para todos os $B \in \mathcal{F}_{\{0, ., n\}}, C \in \mathcal{F}_{\{n \geq 0\}}$. Portanto, posto que $A$ é invariante e escolhendo $B=C=A$, temos que 


$$
\mathbb{P}\{A\}=\lim _{l \rightarrow \infty} \mathbb{P}\left\{A \cap T^{-(n+l+1)} A\right\}=\mathbb{P}\{A\}^{2}
$$

então, $\mathbb{P}\{A\}=0$ ou $\mathbb{P}\{A\}=1$. Isto para todo $A \in \mathcal{F}_{\{0, ., n\}}$. Agora, para $A \in \mathcal{F}$ qualquer e para todo $\epsilon>0$ existem $n$ natural e $A^{\prime} \in \mathcal{F}_{\{0, ., n\}}$ tais que $\mathbb{P}\left\{A \triangle A^{\prime}\right\}<\epsilon$. Notemos que agora vale (1.2) para $A^{\prime}$. Por outro lado, existe um $k$ inteiro positivo tal que

$$
\begin{aligned}
\left|\mathbb{P}\{A\}-\mathbb{P}\left\{A^{\prime} \cap T^{-k} A^{\prime}\right\}\right| & =\left|\mathbb{P}\left\{A \cap T^{-k} A\right\}-\mathbb{P}\left\{A^{\prime} \cap T^{-k} A^{\prime}\right\}\right| \\
& \leq\left|\mathbb{P}\left\{\left(A \cap T^{-k} A\right) \triangle\left(A^{\prime} \cap T^{-k} A^{\prime}\right)\right\}\right| \\
& \leq 2 \mathbb{P}\left\{A \triangle A^{\prime}\right\} \leq 2 \epsilon .
\end{aligned}
$$

Pela mistura uniforme podemos escolher um $k$ tal que

$$
\left|\mathbb{P}\left\{A^{\prime} \cap T^{-k} A^{\prime}\right\}-\mathbb{P}\left\{A^{\prime}\right\}^{2}\right| \leq \epsilon
$$

Por último

$$
\left|\mathbb{P}\left\{A^{\prime}\right\}^{2}-\mathbb{P}\{A\}^{2}\right| \leq 2 \epsilon
$$

Logo $\left|\mathbb{P}\{A\}-\mathbb{P}\{A\}^{2}\right|<5 \epsilon$. Posto que $\epsilon$ é arbitrário, $\mathbb{P}\{A\}=0$ ou $\mathbb{P}\{A\}=1$.

\subsubsection{Exemplos}

Para estas definições fazerem sentido, é preciso mostrar que estas familias são não vazias e que realmente diferem uma da outra.

Exemplo 1.15 O primeiro exemplo básico é uma sequência de variáveis aleatórias de Bernoulli independentes. Neste caso, o processo é $\phi$-misturador com função $\phi \equiv 0$. 
Exemplo 1.16 O segundo natural exemplo natural é o de uma cadeia de Markov irredutível e aperiódica com espaço de estados finito. Neste caso, a cadeia é um processo $\phi$-misturador com função $\phi$ dada por $\phi(n) \leq e^{-c n}$ com constante c positiva. Veremos abaixo a prova desse fato.

Prova. Sejam $A=\left\{X_{1}^{n}=a_{1}^{n}\right\}$ um $n$-cilindro e $B=\left\{X_{n+l+1}^{n+l+m}=b_{1}^{m}\right\}$ um $m$-cilindro. Então, pela propriedade markoviana

$$
\begin{aligned}
& |\mathbb{P}\{A \cap B\}-\mathbb{P}\{A\} \mathbb{P}\{B\}| \\
= & \left|\mathbb{P}\{A\} \frac{\mathbb{P}\left\{X_{n+l+1}=b_{1} \mid X_{n}=a_{n}\right\}}{\mathbb{I P}\left\{X_{n+l+1}=b_{1}\right\}} \mathbb{P}\{B\}-\mathbb{P}\{A\} \mathbb{P}\{B\}\right| \\
\leq & \mathbb{P}\{A\} \mathbb{P}\{B\} \frac{\mathcal{D}^{l}}{\rho},
\end{aligned}
$$

onde $0<\mathcal{D}<1$ é o coeficiente de ergodicidade de Dobrushin (ver por exemplo, Teorema 3.23 em Ferrari e Galves (1997)) e $\rho=\inf _{b \in \mathcal{E}} \mathbb{P}\{b\}$.

Sejam agora $A \in \mathcal{F}_{\{0, ., n\}}, B \in \mathcal{F}_{\{n+l+1, ., n+l+m\}}$ então, podemos escrever $A=\cup_{i \in I} A_{i}$ e $B=\cup_{j \in J} B_{j}$ onde os $A_{i}$ são $n$-cilindros e os $B_{i}$ são $m$-cilindros e as uniões são disjuntas.

$$
\begin{aligned}
|\mathbb{P}\{A \cap B\}-\mathbb{P}\{A\} \mathbb{P}\{B\}| & =\left|\mathbb{P}\left\{\bigcup_{i \in I} A_{i} \cap \bigcup_{j \in J} B_{j}\right\}-\mathbb{P}\left\{\bigcup_{i \in I} A_{i}\right\} \mathbb{P}\left\{\bigcup_{j \in J} B_{j}\right\}\right| \\
& =\sum_{i \in I, j \in J}\left|\mathbb{P}\left\{A_{i} \cap B_{j}\right\}-\mathbb{P}\left\{A_{i}\right\} \mathbb{P}\left\{B_{j}\right\}\right| \\
& \leq \sum_{i \in I, j \in J}\left|\mathbb{P}\left\{A_{i}\right\} \mathbb{P}\left\{B_{j}\right\}\right| \frac{\mathcal{D}^{l}}{\rho} \\
& =\mathbb{P}\{A\} \mathbb{P}\{B\} \frac{\mathcal{D}^{l}}{\rho} .
\end{aligned}
$$

Isto conclui a prova.

Exemplo 1.17 Toda fonte gibbsiana com potencial Hölder contínuo é um processo $\phi$-misturador com $\phi(n) \leq e^{-c n}$. Referimos o leitor a Bowen (1975) para definições e propriedades sobre este tipo de processos. 
Exemplo 1.18 Uma cadeia com conexões completas, seguindo a definição de Bressaud, Fernandez e Galves (1999), é um processo que satisfaz as seguintes propriedades

1. para todo $a_{1}, \ldots, a_{n} \in \mathcal{E}$,

$$
\mathbb{P}\left\{X_{1}=a_{1}, \ldots, X_{n}=a_{n}\right\}>0
$$

2. o limite

$$
\lim _{m \rightarrow \infty} \mathbb{P}\left\{X_{0}=a_{0} \mid X_{j}=a_{j},-m \leq j \leq-1\right\}=\mathbb{P}\left\{X_{0}=a_{0} \mid X_{j}=a_{j}, j \leq-1\right\}
$$

existe para todo $a_{j}, j \leq-1$,

3. existe uma sequência $\left\{\gamma_{m}\right\}_{m \geq 1}$ com $\gamma_{m} \leq C e^{-c m}$, tal que, para todo $a_{j}, b_{j} \in A, j \leq-1$ com $a_{j}=b_{j}$ for $-1 \geq j \geq-m$,

$$
\left|\frac{\mathbb{P}\left\{X_{0}=a_{0} \mid X_{j}=a_{j}, j \leq-1\right\}}{\mathbb{P}\left\{X_{0}=a_{0} \mid X_{j}=b_{j}, j \leq-1\right\}}-1\right| \leq \gamma_{m} .
$$

Toda fonte gibbsiana com potencial Hölder contínuo é uma cadeia com conexões completas. A propriedade 2 é garantida pela continuidade do potencial. A propriedade 3 é garantida pela condição Hölder. Por outro lado, toda cadeia com conexoes completas é um processo $\alpha-$ misturador. (Ver Schmitt, (2000).)

Exemplo 1.19 Haydn e Vaienti (2000) estudam os processos chamados de $(\phi-f)$-misturadores. Estes podem ser descritos informalmente como processos $\phi$-misturadores que possuem uma função $f$ que especifica uma minoração para o comprimento do buraco entre os eventos $B$ e $C$ para que o processo possua propriedade de mistura. Mais precisamente, um processo é dito $(\phi-f)$-misturador se existe uma função $f: \bigcup_{k \geq 1} \mathcal{E}^{k} \rightarrow \mathbb{N}_{0}$ crescente respeito do comprimento do cilindro $B \subseteq \Omega$, tal que a desigualdade (1.1) é satisfeita toda vez que $l \geq f(B)$. Esses processos podem ser vistos então, como uma generalização dos processos $\phi$-misturadores e somente são $\phi$-misturadores quando $f \equiv$ constante.

Concluímos esta seção com algumas referências.

O livro de Doukhan (1995) é uma importante fonte de definições, exemplos e referencias para construir exemplos das diferentes familias acima definidas que incluem processos uniformemente misturadores não $\alpha$-misturadores; processos $\alpha$-misturadores não $\phi$-misturadores, e processos $\phi$-misturadores com função decrescente $\phi$ qualquer.

O livro de Shields (1996) é uma boa introdução aos processos misturadores e à Teoria Ergódica. 


\section{Capítulo 2}

\section{RESULTADOS PRÉVIOS}

Apresentaremos neste capítulo, alguns resultados prévios no estudo dos instantes de entrada e instantes de volta de um evento raro em processos misturadores. $\mathrm{O}$ estudo comparativo entre eles nos permitirá dizer quais são os pontos importantes a serem resgatados de cada resultado, assim como, prantear algumas perguntas que tentaremos responder nos próximos capítulos.

\subsection{Galves e Schmitt 1997}

Este trabalho considera o problema do tempo de entrada em um evento raro no marco geral de processos $\phi$-misturadores com função somável $\phi$. Um ponto chave é que o resultado aqui apresentado, que prova a convergência da lei do instante de entrada para a lei exponencial, é valido para todo tipo de cilindro. O fator de escala na lei exponencial é $\lambda_{A} \mathbb{P}\{A\} \operatorname{com} \lambda_{A}$ pertencendo a um intervalo limitado longe do zero e do infinito. O resultado é o seguinte

Teorema 2.1 (Galves e Schmitt, 1997) Seja $\left\{X_{m}\right\}_{m \in \mathbb{Z}}$ um processo $\phi$-misturador e suponhamos que a função $\phi: \mathbb{I N} \rightarrow \mathbb{R}_{+}$é somável. Então, existem quatro constantes estritamente positivas $\Lambda_{1}, \Lambda_{2}, \beta, C$, tais que para todo $n$ e todo $A \in \mathcal{C}_{n}$, existe $\lambda(A) \in\left[\Lambda_{1}, \Lambda_{2}\right]$, para os quais vale a seguinte desigualdade

$$
\sup _{t>0}\left|\mathbb{P}\left\{\tau_{A}>\frac{t}{\lambda(A) \mathbb{P}\{A\}}\right\}-e^{-t}\right| \leq C \mathbb{P}\{A\}^{\beta}
$$

Uma questão natural sugerida pelo teorema é a de precisar o valor do parâmetro $\lambda_{A}$. O Lema de Kac sugere que $\lambda_{A}=1$. Collet, Galves e Schmitt (1999) mostram que este é tipicamente o caso. 


\subsection{Collet, Galves e Schmitt 1999}

Collet, Galves e Schmitt (1999) mostram que o fator de escala $\lambda_{A}$ depende da volta do evento raro sobre ele mesmo nos instantes curtos de tempo. Como consequência, para a maioria dos cilindros o fator de escala $\lambda_{A}$ em (2.1) é assintoticamente um, como é sugerido pelo Lema de Kac.

Definição 2.2 Seja $s \in \mathbb{I N}$. Definamos $\mathcal{B}_{n}$ como o conjunto das sequências de comprimento $n$ de elementos em $\mathcal{E}$ que recorrem antes do instante $n / 3$. Ou seja, se $A \in \mathcal{C}_{n}$, diremos que $A \in \mathcal{B}_{n}$ se e somente se existe um inteiro $j, 1 \leq j \leq n / 3$ tal que $A \cap T^{-j} A \neq \emptyset$.

Teorema 2.3 (Collet, Galves e Schmitt, 1999) Seja $\left\{X_{m}\right\}_{m \in \mathbb{Z}}$ um processo $\phi$-misturador e suponhamos que a função $\phi: \mathbb{I N} \rightarrow \mathbb{R}_{+}$é exponencial. Então, existem duas constantes estritamente positivas $c, C$, tais que para todo $n$ e todo $A \in \mathcal{C}_{n} \backslash \mathcal{B}_{n}$, vale a seguinte desigualdade

$$
\sup _{t>0}\left|\mathbb{P}\left\{\tau_{A}>\frac{t}{\mathbb{P}\{A\}}\right\}-e^{-t}\right| \leq C e^{-c n}
$$

Mais ainda,

$$
\mathbb{I P}\left\{\mathcal{B}_{n}\right\} \leq C e^{-c n}
$$

\subsection{Hirata, Saussol e Vaienti 1999}

Este trabalho mostra que a distribuição do instante de entrada num evento $A$ e o instante de volta no $A$, re-escalados adequadamente, estão próximos de uma lei exponencial de parâmetro um, se e somente, se ambas as distribuições estão mutuamente próximas no sentido seguinte,

Teorema 2.4 (Hirata, Saussol e Vaienti, 1999) Definamos $c(k, A)=\mid \mathbb{P}_{A}\left(\tau_{A}>k\right)-\mathbb{P}\left(\tau_{A}\right\rangle$ $k) \mid$ e chamemos $c(A)=\sup _{k} c(k, A)$. A distribuição re-escalada do instante de entrada no conjunto $A$ difere da lei exponencial de parâmetro um no máximo por $d(A):=4 \operatorname{IP}\{A\}+c(A)(1+$ $\left.\log c(A)^{-1}\right)$, isto é

$$
\sup _{t \geq 0}\left|\mathbb{P}\left\{\tau_{A}>\frac{t}{\mathbb{P}\{A\}}\right\}-e^{-t}\right| \leq d(U) .
$$


E o mesmo vale começando em $A$

$$
\sup _{t \geq 0}\left|\mathbb{P}_{A}\left\{\tau_{A}>\frac{t}{\mathbb{P}\{A\}}\right\}-e^{-t}\right| \leq d(U)
$$

Conversamente, a diferença entre o instante de entrada (re-escalado) e o instante de volta (reescalado) pode ser majorada em termos da distáncia $\bar{c}(A):=\sup _{t \geq 0}\left|\mathbb{P}_{A}\left\{\tau_{A}>t / \mathbb{P}\{A\}\right\}-e^{-t}\right|$, isto é,

$$
c(A) \leq 2 \mathbb{P}\{A\}+\bar{c}(A)\left(2+\log \bar{c}(A)^{-1}\right) .
$$

Este teorema provê uma condição necessária e suficiente para obter a lei exponencial para o instante de entrada e o instante de volta a um evento raro. Isto é, $c(A) \rightarrow 0$, se e somente se, $\bar{c}(A) \rightarrow 0$. A prova é valida para uma medida estacionária $\mathbb{I P}$ qualquer, sem assumir sequer ergodicidade. É claro que, nessa generalidade, não se pode esperar a convergência de $c(A)$. $\mathrm{O}$ seguinte lema oferece uma majoração para $c(A)$, e além disso, mostra quando pode-se esperar a convergência.

Lema 2.5 Seja $A \subset \Omega$ um conjunto mensurável. Então, se satisfaz a seguinte desigualdade

$$
c(A) \leq \inf _{k \in N}\left\{a_{k}(A)+b_{k}(A)+k \mathbb{P}\{A\}\right\}
$$

onde as quantidades são definidas por

$$
\begin{aligned}
& a_{k}(A)=\mathbb{P}_{A}\left\{\bigcup_{j=1}^{k} T^{-j} A\right\}=\mathbb{P}_{A}\left\{\tau_{A} \leq k\right\}, \\
& b_{k}(A)=\sup _{W \in \mathcal{F}_{\{0, ., n\}}}\left|\mathbb{P}_{A}\left\{T^{-k} W\right\}-\mathbb{P}\{W\}\right| .
\end{aligned}
$$


Note-se que, para todo $k \geq n, b_{k}(A)=\alpha(k-n)$, se o processo é $\alpha$-misturador, e $b_{k}(A)=$ $\gamma(k-n) / \mathbb{P}\{A\}$, se o processo é $\gamma$-misturador.

O elemento chave nesta majoração é a quantidade $a_{k}(A)=\mathbb{P}_{A}\left\{\tau_{A} \leq k\right\}$. Por exemplo, se $A \in \mathcal{C}_{n} \backslash \mathcal{B}_{n}$, então $a_{k}(A)=0$ para $k=1, \ldots, n / 3$. Se o processo perde memória suficientemente rápido, então $b_{k}(A)$ tende a 0 e o teorema garante a convergência assintótica para a lei exponencial.

É importante observar que em situações várias $c(A)$ não converge a zero. O modelo de Ehrenfest clássico provê um exemplo desse fato.

Exemplo 2.6 O modelo de Ehrenfest é uma cadeia de Markov, tomando valores no conjunto $\mathcal{E}=\{0,1\}^{N}$, onde $N$ é um inteiro positivo. Para qualquer $x \in \mathcal{E}$ e qualquer índice $i \in\{1, \ldots, N\}$, o estado $x^{i}$ é definido como a sequência que coincide com $x$ em todas as coordenadas com exceção da coordenada i-ésima. Definimos as probabilidades de transição da cadeia como segue

$$
Q(x, y)=\left\{\begin{aligned}
\frac{1}{2 N} \quad \text { se } y=x^{i}, \text { para algum } i=1, \ldots, N \\
\frac{1}{2} \quad \text { se } y=x \\
0 \quad \text { caso contrario } .
\end{aligned}\right.
$$

A medida invariante da cadeia é a distribuição uniforme sobre $\mathcal{E}$. Agora, consideramos o evento $A=\{(1, \ldots, 1)\}$. Se $N$ é suficientemente grande este é um evento raro. Suponhamos que a cadeia começa com sua medida invariante e definamos $\tau_{A}$ como o primeiro instante que a cadeia visita o evento $A$.

Por um cálculo direto,

$$
\mathbb{P}_{A}\left\{\tau_{A}>1\right\}=\frac{1}{2}
$$




$$
\mathbb{P}\left\{\tau_{A}>1\right\} \geq 1-\frac{N+1}{2 N}
$$

Portanto, $c(1, A)$ converge a $1 / 2$, quando $N$ diverge.

Hirata, Saussol e Vaienti (1999) e Collet, Galves e Schmitt (1999) demonstram que $\mathbb{I P}\{A\}$ é o correto fator de escala para cilindros que não recorrem demasiado rápido. Galves e Schmitt (1998) provam que a aproximação exponencial é valida para todo cilindro usando o fator de escala $\lambda_{A} \mathbb{P}\{A\}$, onde a possibilidade $\lambda_{A}=1$ não é excluída.

Os trabalhos acima mencionados deixam aberta a questão sobre o valor correto de $\lambda_{A}$ para cilindros que recorrem. É certo que ele pode ser diferente de 1? Em Asselah e Dai Pra (1997 a e b) a questão é respondida para o processo de exclusão simples simétrico e para um sistema unidimensional spines independentes, quando $\tau$ é a primeira vez que a densidade empírica em uma caixa grande excede seu valor de equilíbrio. Em ambos os casos $\lambda_{A} \neq 1$.

No próximo capítulo provaremos que a mesma situação ocorre no quadro geral de processos misturadores e apresentaremos uma estimativa calculável desse parâmetro. 


\section{Capítulo 3}

\section{INSTANTE DE ENTRADA}

Dedicaremos este capítulo à majoração e minoração do erro na aproximação da lei do instante de entrada em um evento raro pela lei exponencial. Veremos também algumas consequências desses resultados.

\subsection{Majoração}

Até o momento, os trabalhos na área mostravam primeiro a convergência assintótica da lei do tempo de espera de ocorrência de um evento raro $A$ para uma lei exponencial sem velocidade de convergência e depois com velocidade de convergência em função da esperança do evento, $\mathbb{E}(A)$, ou da medida do evento, $\mathbb{P}\{A\}$. Para estimar a lei do tempo de entrada usando a lei exponencial, esta aproximação é interesante para valores de $t$ próximos de $1 / \mathbb{P}\{A\}$, mas é sem interesse como meio para prover uma real estimativa da lei para valores de $t$ muito grandes onde o valor da lei é muito menor que o valor do erro na aproximação.

Mostraremos assim, uma majoração do erro na aproximação exponencial em função de $t$ e veremos que tipo de conclusões pode se extrair dali.

\subsubsection{Resultados}

Teorema 3.1 Seja $\left\{X_{m}\right\}_{m \in \mathbb{Z}}$ um processo estocástico estacionário $\phi$-misturador qualquer ou $\alpha$-misturador com função $\alpha$ somável. Existem constantes estritamente positivas $C, \Lambda_{1}$ e $\Lambda_{2}$ tais que para qualquer $n \in \mathbb{I N}$ e $A \in \mathcal{C}_{n}$, existem constantes $\widetilde{\lambda_{A}}, \delta_{A}$ com $\Lambda_{1} \leq \widetilde{\lambda_{A}}, \delta_{A} \leq \Lambda_{2}$. para as quais as seguintes desigualdades são verdadeiras para todo $t>0$. 


\section{A convergência assintótica}

$$
\left|\mathbb{P}\left\{\tau_{A}>t\right\}-e^{-\widetilde{\lambda_{A}} P\{A\} t}\right| \leq C\left[\mathbb{P}\{A\}^{\beta+1} t+\mathbb{P}\{A\}^{\beta}\right] e^{-\widetilde{\lambda_{A}} P\{A\} t}
$$

com $0<\beta<1$.

\section{A majoração da cauda da distribuição}

$$
\left|\mathbb{P}\left\{\tau_{A}>t\right\}-e^{-t \delta_{A} P\{A\}}\right| \leq C[\mathbb{P P}\{A\} t] e^{-\delta_{A} P\{A\} t}
$$

Da primeira parte do teorema se desprende a convergência uniforme respeito da medida do evento, $\mathbb{P}\{A\}$, pois o máximo com respeito a $t$, do membro da direita em (3.1) é alcançado em $t=1 / \mathbb{P}\{A\}$, e vale $C \mathbb{P}\{A\}^{\beta}$.

Embora a segunda parte não mostre a convergência uniforme (pois o máximo em função de $t$ do erro é uma constante que não depende de $I P\{A\}$ ), ela fornece, em principio, um controle sobre o decaimento da cauda da distribuição melhor do que o dado pela parte 1 do teorema.

No resto desta seção, a menos que seja explicitamente estabelecido o contrário, assumiremos as hipóteses do presente teorema. Quando uma propriedade valer para processos $\alpha$ ou $\phi$ misturadores, escreveremos simplesmente *.

Estabeleceremos agora, o teorema principal deste capítulo.

Teorema 3.2 Para quaisquer $n \in \mathbb{I N}, A \in \mathcal{C}_{n}$, existe uma constante $\xi_{A} \in\left[\Lambda_{1}, \Lambda_{2}\right]$, tal que vale a seguinte desigualdade

$$
\left|\mathbb{P}\left\{\tau_{A}>t\right\}-e^{-\xi_{A} P\{A\} t}\right| \leq C \Delta \mathbb{P}\{A\}[\mathbb{P}\{A\} t \vee 1] e^{-\xi_{A} P\{A\} t},
$$

onde $\Delta$ é o valor que realiza $o \inf _{n \leq \Delta \leq \frac{1}{2} \frac{1}{P\{A\}}}[\Delta \mathbb{P}\{A\}+*(\Delta)]$.

É imediato um corolário que diz qual a velocidade de convergência uniforme da distribuição de $\tau_{A}$ para a lei exponencial. 
Corolário 3.3 Vale a seguinte desigualdade

$$
\sup _{t>0}\left|\mathbb{P}\left\{\tau_{A}>t\right\}-e^{-\xi_{A} P\{A\} t}\right| \leq C \Delta \mathbb{P}\{A\}
$$

onde $\Delta$ é o mesmo do Teorema 3.2.

Prova. O máximo respeito de $t$ do membro da direita em (3.2) é alcançado em $t=1 / \mathbb{P}\{A\}$, e vale $C \triangle I P\{A\}$.

Antes de apresentar as provas, nas próximas três seções, veremos algumas propriedades que nos ajudarão na demonstração.

\subsubsection{Estimação da medida de um cilindro}

Uma propriedade importante dos processos misturadores é o decaimento exponencial da medida dos cilindros que será provado no próximo lema. Isto pode ser visto como uma espécie de versão groseira do Teorema de Shanon-Mc Millan-Breiman.

Lema 3.4 Seja um processo $\alpha$-misturador com função $\alpha$ decrescente qualquer. Existem constantes estritamente positivas $C$ e $\Gamma$ tais que para todo inteiro positivo $n$, e para todo cilindro $A \in \mathcal{C}_{n}$, vale a seguinte desigualdade

$$
\mathbb{I P}\{A\} \leq C e^{-\Gamma n}
$$

Prova. Escrevamos $A=\left\{X_{0}^{n-1}=a_{0}^{n-1}\right\}$. Fixemos um inteiro positivo $n_{0}$. Suponhamos que $n>n_{0}$ e que $n=q n_{0}+r$, com $q$ inteiro positivo e $r$ inteiro $0 \leq r<n_{0}$. Observamos primeiro que

$$
\mathbb{P P}\left\{X_{0}^{n-1}=a_{0}^{n-1}\right\} \leq \mathbb{P}\left\{X_{0}=a_{0}, X_{n_{0}}=a_{n_{0}}, \ldots, X_{(q-1) n_{0}}=a_{(q-1) n_{0}}\right\}
$$

Usando iteradamente a propriedade $\alpha$-misturadora temos 


$$
\mathbb{I P}\{A\} \leq\left[\alpha\left(n_{0}-1\right)+\rho\right]^{\left[\frac{n}{n_{0}}\right]},
$$

onde $[x]$ denota a parte inteira de $x$ e

$$
\rho=\sup \left\{\mathbb{P}\left(a_{i}\right): a_{i} \in \mathcal{E}\right\}
$$

Posto que, por hipótese, $\rho<1$ e a função $\alpha(l) \rightarrow 0$, podemos escolher o inteiro $n_{0}$ tal que

$$
\left(\rho+\alpha\left(n_{0}-1\right)\right)<1
$$

Isto prova o decaimento exponencial para todo $n$-cilindro com $n \geq n_{0}$. Mudando a constante $C$ provamos (3.3) para qualquer $n$.

É importante observar que o decaimento exponencial dos cilindros é uma propriedade que garante ao processo possuir entropia positiva.

Proposição 3.5 Seja $\left\{X_{m}\right\}_{m \in \mathbb{Z}}$ um processo ergódico qualquer tal que $\mathbb{P}\left\{A_{n}\right\} \leq C e^{-\Gamma n}$, para todo $n$ natural e para todo $n$-cilindro $A_{n}$, com constantes positivas $C$ e $\Gamma$. Então o processo possui entropia positiva. Mais ainda, $h \geq \Gamma$.

Prova. A hipótese implica que

$$
\log \mathbb{I P}\left\{A_{n}\right\} \leq \log C-\Gamma n
$$

portanto, pelo Teorema de Shannon-Mc Millan-Breinman, e para quase todo $\omega \in \Omega$,

$$
h=-\lim _{n \rightarrow \infty} \frac{1}{n} \log \mathbb{P}\left\{A_{n}(\omega)\right\} \geq-\lim _{n \rightarrow \infty}\left[\frac{\log C}{n}-\Gamma\right]=\Gamma>0,
$$


onde $A_{n}(\omega)$ é o $n$-cilindro de coordenadas $\omega_{0}, \ldots, \omega_{n-1}$.

Isto conclui a prova da proposição.

O próxima lema estuda o comportamento do processo em torno de uma vizinhança de tamanho $n$ de um $n$-cilindro $A$. Ele diz, informalmente, que quando um processo perde memória suficientemente rápido, o evento pode voltar dentro da vizinhança um número fixo de vezes e não uma quantidade de ordem $n$ de vezes.

Lema 3.6 Existe uma constante estritamente positiva $C^{\prime}$ tal que para todo inteiro positivo $n$, e para todo cilindro $A \in \mathcal{C}_{n}$, vale a seguinte desigualdade

$$
\sum_{k=1}^{n} \mathbb{P}\left\{A \cap T^{-k} A\right\} \leq C^{\prime} \mathbb{P}\{A\}
$$

Prova. Para provar (3.4), no caso $\alpha$-misturador notamos que

$$
\begin{aligned}
\mathbb{P}\left\{A \cap T^{-k} A\right\} & \leq \mathbb{P}\left\{A \cap T^{-k} A^{(k)}\right\} \leq \mathbb{P}\left\{A \cap T^{-k+\frac{k}{2}} A^{\left(k-\left[\frac{k}{2}\right]\right)}\right\} \\
& \leq \alpha\left(\left[\frac{k}{2}\right]\right) \mathbb{P}\{A\}+\mathbb{P}\left\{A^{\left(k-\left[\frac{k}{2}\right]\right)}\right\} \mathbb{P}\{A\},
\end{aligned}
$$

onde $A^{(l)}=\left\{X_{n-l}^{n-1}=a_{n-l}^{n-1}\right\}$ para todo $l$ natural, $l=1, \ldots, n$. Então, posto que $\alpha$ é somável e usando (3.3)

$$
\sum_{k=1}^{n} \mathbb{P}\left\{A \cap T^{-k} A\right\} \leq 2 \mathbb{P}\{A\} \sum_{k=0}^{\left[\frac{n}{2}\right]} \alpha(k)+2 \mathbb{P}\{A\} \sum_{k=1}^{n-\left[\frac{n}{2}\right]} \mathbb{P}\left\{A^{(k)}\right\} \leq C^{\prime} \mathbb{P}\{A\}
$$

Isto prova o caso $\alpha$-misturador. No caso $\phi$-misturador geral enviamos o leitor ao Lema 1 em Galves e Schmitt (1997). 


\subsubsection{O parâmetro de re-escalamento}

Para um inteiro positivo $k$ definimos

$$
N_{k}=\sum_{l=1}^{k} \mathbb{1}_{T^{-l}(A)}
$$

onde $\mathbb{1}_{A}$ é a função indicadora do conjunto $A$. Para uma realização do processo $\omega \in \Omega, N_{k}(\omega)$ é o número de vezes que o processo visita $A$, durante os primeiros $k$ pasos. Observamos que

$$
\left\{\tau_{A} \leq k\right\}=\left\{N_{k} \geq 1\right\}
$$

Lema 3.7 Seja $\left\{X_{m}\right\}_{m \in \mathbb{Z}}$ um processo estacionario qualquer. Para todo número real positivo $t \geq 1$ a seguinte desigualdade é verdadeira

$$
\mathbb{P}\left\{\tau_{A} \leq t\right\} \leq t \mathbb{P}\{A\}
$$

\section{Prova.}

$$
\mathbb{P}\left\{\tau_{A} \leq t\right\}=\sum_{l=1}^{[t]} \mathbb{P}\left\{\tau_{A}=l\right\} \leq \sum_{l=1}^{[t]} \mathbb{P}\left\{T^{-l} A\right\}=[t] \mathbb{P}\{A\}
$$

onde na última desigualdade usamos a invariança da medida $\mathbb{P}$ com respeito à transformação $T$. 
Lema 3.8 Para todo inteiro positivo $n$, todo cilindro $A \in \mathcal{C}_{n}$ e todo $t>0$, vale a seguinte desigualdade

$$
\mathbb{I P}\left\{\tau_{A} \leq t\right\} \geq \frac{[t]^{2} \mathbb{P}\{A\}^{2}}{t \mathbb{P}\{A\}+C^{\prime} t \mathbb{P}\{A\}+C(t \mathbb{P}\{A\})^{2}+2 K t \mathbb{P}\{A\}}
$$

onde $C^{\prime}>0, C>0$ e $K>0$ são constantes independentes de $n$ e A.

Prova. Escrevamos por simplicidade $N=N_{[t]}$.

Primeiro observamos que

$$
(\mathbb{E}(N))^{2}=\left(\mathbb{E}\left(N \mathbb{1}_{\{N \geq 1\}}\right)\right)^{2} \leq \mathbb{E}\left(N^{2}\right) \mathbb{P}\{N \geq 1\}
$$

onde a última desigualdade segue da desigualdade de Schwarz.

Pela equação (3.6) temos

$$
\mathbb{P}\left\{\tau_{A} \leq t\right\}=\mathbb{P}\{N \geq 1\} \geq \frac{(\mathbb{E}(N))^{2}}{\mathbb{E}\left(N^{2}\right)}
$$

Precisamos controlar então $\mathbb{E}(N)$ e $\mathbb{E}\left(N^{2}\right)$. Por definição $\mathbb{E}(N)=[t] \mathbb{P}\{A\}$.

Para obter uma majoração do denominador $\mathbb{E}\left(N^{2}\right)$, decompomo-lo da seguinte maneira. Assumimos primeiro que $[t]>n$. A definição de $N$ nos diz que

$$
\begin{aligned}
\mathbb{E}\left(N^{2}\right)=\sum_{l=1}^{[t]} \mathbb{E}\left(\mathbb{1}_{T^{-l}(A)}\right) & +2 \sum_{l=1}^{n}([t]-l) \mathbb{E}\left(\mathbb{1}_{A} \mathbb{I}_{T^{-l}(A)}\right) \\
& +2 \sum_{l=n+1}^{[t]-1}([t]-l) \mathbb{E}\left(\mathbb{I}_{A} \mathbb{I}_{T^{-l}(A)}\right) .
\end{aligned}
$$


O primeiro termo desta decomposição é $\mathbb{E}(N)=[t] \mathbb{P}\{A\}$.

Usando a equação (3.4) no segundo termo, obtemos

$$
\sum_{l=1}^{n}([t]-l) \mathbb{E}\left(\mathbb{1}_{A} \mathbb{1}_{T^{-l}(A)}\right) \leq[t] \sum_{l=1}^{n} \mathbb{P}\left\{A \cap T^{-l}(A)\right\} \leq C^{\prime} t \mathbb{P}\{A\}
$$

A propriedade $\alpha$-misturadora provê majorações para a esperança no terceiro termo.

$$
\mathbb{E}\left(\mathbb{1}_{A} \mathbb{1}_{A}\left(T^{l}\right)\right) \leq \mathbb{P}\{A\}^{2}+\alpha(l-n-1) \mathbb{P}\{A\}
$$

Portanto o terceiro termo é majorado por

$$
2 \mathbb{P}\{A\}\left(\mathbb{P}\{A\} \sum_{l=1}^{[t]-1} l+t \sum_{l=1}^{+\infty} \alpha(l)\right)
$$

Agora, observamos que

$$
\sum_{l=1}^{[t]-1} l=\frac{1}{2}[t]([t]-1)
$$

Finalmente, usamos a hipótese que a série $\sum_{l=1}^{+\infty} \alpha(l)$ é somável para obter a majoração

$$
2 \sum_{l=n+1}^{[t]}([t]-l) \mathbb{E}\left(\mathbb{I}_{A} \mathbb{I}_{T^{-l}(A)}\right) \leq(t \mathbb{P}\{A\})^{2}+2 K t \mathbb{I P}\{A\}
$$

onde $K=\sum_{l=1}^{+\infty} \alpha(l)<+\infty$. 
No caso $\phi$-misturador simplesmente observamos que

$$
\mathbb{E}\left(\mathbb{I}_{A} \mathbb{1}_{T^{-l}(A)}\right) \leq(1+\phi(l-n)) \mathbb{P}\{A\}^{2}
$$

Portanto, o terceiro termo é majorado por

$$
2 \frac{1}{2}[t]([t]-1)(1+\phi(0)) \mathbb{P}\{A\}^{2} \leq C(t \mathbb{P}\{A\})^{2} .
$$

Para $t \leq n$ a prova é a mesma (com o terceiro termo na equação (3.11) ausente). Isto conclui a prova do lema.

Seja $f_{A}$ um inteiro positivo (dependendo de $A$ ) tal que

$$
f_{A} \mathbb{P}\{A\}<\frac{1}{2}
$$

\section{Definimos}

$$
\lambda_{A, f}=\frac{-\log \mathbb{I P}\left\{\tau_{A}>f_{A}\right\}}{f_{A} I P\{A\}} .
$$

Lema 3.9 Existe um inteiro positivo $n_{0}$, tal que, para todo $n \geq n_{0}$ e $A \in \mathcal{C}_{n}$, as seguintes desigualdades são verdadeiras

$$
\Lambda_{1} \leq \lambda_{A, f} \leq \Lambda_{2}
$$

onde $\Lambda_{1}$ e $\Lambda_{2}$ são duas constantes positivas independentes de $n, A$ e $f_{A}$. 
Prova. Para obter a minoração, primeiro observamos que

$$
\frac{\theta}{2} \leq 1-e^{-\theta} \leq \theta
$$

onde a desigualdade da direita é valida para todo $\theta \geq 0$ e a da esquerda é valida para todo $\theta \in[0,1]$ ou equivalentemente para os $\theta$ tais que $1-e^{-\theta} \leq 1-e^{-1}(>1 / 2)$. Tomemos $\theta=\theta(A)=-\log \mathbb{P}\left\{\tau_{A}>f_{A}\right\}$ e observemos que $\mathbb{I P}\left\{\tau_{A} \leq f_{A}\right\}=1-e^{-\theta}$. Usando o lado direito da equação (3.14) e o Lema 3.8 , temos

$$
\begin{aligned}
\frac{-\log \mathbb{P}\left\{\tau_{A}>f_{A}\right\}}{f_{A} \mathbb{P}\{A\}} & \geq \frac{\mathbb{P}\left\{\tau_{A} \leq f_{A}\right\}}{f_{A} \mathbb{P}\{A\}} \geq \frac{1}{1+C^{\prime}+C f_{A} \operatorname{IP}\{A\}+2 K} \\
& \geq \frac{1}{1+C^{\prime}+C / 2+2 K}=\Lambda_{1}
\end{aligned}
$$

onde $C^{\prime}, C$ e $K$ são as mesmas constantes que aparecem no Lema 3.8 . que

Por outro lado, se segue das equações (3.12), (3.7) e do lado esquerdo da equação (3.14),

$$
-\log \mathbb{P}\left\{\tau_{A}>f_{A}\right\} \leq 2 \mathbb{P}\left\{\tau_{A} \leq f_{A}\right\} \leq 2 f_{A} \mathbb{P}\{A\}
$$

para todo $n \geq n_{0}$ e podemos tomar $\Lambda_{2}=2$. Isto conclui a prova do lema.

\subsubsection{Propriedade de independência}

O seguinte lema nos mostra qual é o erro cometido ao fatorar os eventos mensuráveis que determinam a distribuição do instante de entrada num cilindro de comprimento $n$.

Lema 3.10 Seja $\left\{X_{m}\right\}_{m \in \mathbb{Z}}$ um processo $\alpha$-misturador. Seja $A \in \mathcal{C}_{n}$ e $t, s \in \mathbb{I N}$ tais que $t+s>n$. Para todo $\Delta \in \mathbb{I N}$ com $n \leq \Delta \leq t+s$, vale a seguinte desigualdade

$$
\left|\mathbb{P}\left\{\tau_{A}>t+s\right\}-\mathbb{P}\left\{\tau_{A}>t\right\} \mathbb{P}\left\{\tau_{A}>s\right\}\right| \leq 2 \Delta \mathbb{P}\{A\}+\alpha(\Delta-n)
$$


Prova. Usamos a desigualdade triangular. Escolhemos $u>0$, tal que, $t+s \geq u+\Delta$.

$$
\begin{aligned}
& \left|\mathbb{P}\left\{\tau_{A}>t+s\right\}-\mathbb{P}\left\{\tau_{A}>t\right\} \mathbb{P}\left\{\tau_{A}>s\right\}\right| \\
\leq & \left|\mathbb{P}\left\{\tau_{A}>t+s\right\}-\mathbb{P}\left\{\tau_{A}>u \bigcap \tau_{A} \circ T^{u+\Delta}>t+s-u-\Delta\right\}\right| \\
+ & \left|\mathbb{P}\left\{\tau_{A}>u \bigcap \tau_{A} \circ T^{u+\Delta}>t+s-u-\Delta\right\}-\mathbb{P}\left\{\tau_{A}>u\right\} P\left\{\tau_{A}>t+s-u-\Delta\right\}\right| \\
+ & \left|\mathbb{P}\left\{\tau_{A}>u\right\} \mathbb{P}\left\{\tau_{A}>t+s-u-\Delta\right\}-\mathbb{P}\left\{\tau_{A}>t\right\} \mathbb{P}\left\{\tau_{A}>s\right\}\right| .
\end{aligned}
$$

Usamos a invariança da medida e a equação (3.7) para controlar o primeiro termo por $\mathbb{P}\left\{\tau_{A}>u \cap \tau_{A} \circ T^{u} \leq \Delta\right\} \leq \mathbb{P}\left\{\tau_{A} \leq \Delta\right\} \leq \Delta \mathbb{P}\{A\}$.

Usamos a propriedade de mistura para majorar o segundo termo por $\alpha(\Delta-n) \mathbb{P}\left\{\tau_{A}>u\right\}$.

O terceiro é majorado usando a desigualdade triangular, a invariança da medida e a equação (3.7)

$$
\begin{aligned}
& \left|\mathbb{P}\left\{\tau_{A}>u\right\}-\mathbb{P}\left\{\tau_{A}>t\right\}\right| \mathbb{P}\left\{\tau_{A}>t+s-u-\Delta\right\} \\
+ & \mathbb{P}\left\{\tau_{A}>t\right\}\left|\mathbb{P}\left\{\tau_{A}>t+s-u-\Delta\right\}-\mathbb{P}\left\{\tau_{A}>s\right\}\right| \\
\leq & \left|\mathbb{P}\left\{\tau_{A}>u\right\}-\mathbb{P}\left\{\tau_{A}>t\right\}\right|+\mathbb{P}\left\{\tau_{A}>t\right\}\left|\mathbb{P}\left\{\tau_{A}>t+s-u-\Delta\right\}-\mathbb{P}\left\{\tau_{A}>s\right\}\right| \\
\leq & \mathbb{P}\left\{\tau_{A}>u \bigcap \tau_{A} \circ T^{u} \leq t-u\right\}+\mathbb{P}\left\{\tau_{A}>u\right\} \mathbb{P}\left\{\tau_{A} \leq u+\Delta-t\right\} \\
\leq & (t-u) \mathbb{P}\{A\}+(u+\Delta-t) \mathbb{P}\{A\}=\Delta \mathbb{P}\{A\} .
\end{aligned}
$$

Isto conclui a prova do lema.

A seguinte proposição é uma versão iterada do lema anterior.

Proposição 3.11 Seja $\left\{X_{m}\right\}_{m \in \mathbb{Z}}$ um processo $\alpha$-misturador qualquer. Então para todo $f_{A}>$ $n$, existe um $\Delta$ com $n \leq \Delta \leq f_{A} / 2$, tal que para todo $k$ inteiro positivo, valem as seguintes desigualdades

$$
\left|\mathbb{P}\left\{\tau_{A}>k f_{A}\right\}-\mathbb{P}\left\{\tau_{A}>f_{A}-2 \Delta\right\}^{k}\right| \leq C \Delta \mathbb{P}\{A\} k \mathbb{P}\left\{\tau_{A}>f_{A}-2 \Delta\right\}^{k-2}
$$




$$
\left|\mathbb{P}\left\{\tau_{A}>k f_{A}\right\}-\mathbb{P}\left\{\tau_{A}>f_{A}\right\}^{k}\right| \leq C \Delta \mathbb{P}\{A\} k \mathbb{P}\left\{\tau_{A}>f_{A}-2 \Delta\right\}^{k-2}
$$

onde $\Delta$ é o valor que realiza $\inf _{n \leq \Delta \leq \frac{f_{A}}{2}}[\Delta \mathbb{P}\{A\}+\alpha(\Delta)]$.

A diferença entre as equações (3.17) e (3.18) é que na primeira, a geométrica no membro da direita é a mesma que a geométrica no membro da esquerda, enquanto que, na segunda, a geométrica no membro da direita é maior que a geométrica no membro da esquerda, isto é, produz um erro maior. A segunda é tecnicamente mais clara e será usada na prova do Teorema 3.2 .

Prova. Para $k=1$ ambas desigualdades são óbvias. Supohnamos $k \geq 2$. Para cada inteiro no negativo $i$ e para $j=1,2$ denotamos

$$
\mathcal{N}_{j}^{i}=\left\{\tau_{A} \circ T^{i f_{A}+j \Delta}>f_{A}-j \Delta\right\}
$$

Escreveremos também por simplicidade

$$
\mathcal{N}=\left\{\tau_{A}>f_{A}-2 \Delta\right\}
$$

A idéia da demonstração é, usando a desigualdade triangular, iterativamente introduzir um "buraco" de tamanho $\Delta$ na lei do instante de entrada, para logo fatorar usando a propriedade misturadora. Quanto maior for o buraco, maior será a perda de memória mas por outro lado, mais diferirá este novo conjunto da lei de entrada, e vice-versa. De maneira que o jogo consiste em compensar essas duas quantidades.

Primeiramente notamos que, pela estacionaridade da medida, $\mathbb{P}\{\mathcal{N}\}=\mathbb{P}\left\{\mathcal{N}_{0}^{i}\right\}$ para todo $i$ inteiro positivo.

A desigualdade triangular nos dá 


$$
\begin{aligned}
& \left|\mathbb{P}\left\{\tau_{A}>k f_{A}\right\}-\mathbb{P}\{\mathcal{N}\}^{k}\right| \\
\leq & \sum_{j=0}^{k-2} \mathbb{P}\{\mathcal{N}\}^{j}\left|\mathbb{P}\left\{\tau_{A}>(k-j) f_{A}\right\}-\mathbb{P}\left\{\tau_{A}>(k-j-1) f_{A} \bigcap \mathcal{N}_{2}^{k-j-1}\right\}\right| \\
+ & \sum_{j=0}^{k-2} \mathbb{P}\{\mathcal{N}\}^{j}\left|\mathbb{P}\left\{\tau_{A}>(k-j-1) f_{A} \bigcap \mathcal{N}_{2}^{k-j-1}\right\}-\mathbb{P}\left\{\tau_{A}>(k-j-1) f_{A}\right\} \mathbb{P}\left\{\mathcal{N}_{2}^{0}\right\}\right| \\
+ & \mathbb{P}\{\mathcal{N}\}^{k-1}\left|\mathbb{P}\left\{\tau_{A}>f_{A}\right\}-\mathbb{P}\{\mathcal{N}\}\right| .
\end{aligned}
$$

O termo (3.21) é igual a

$$
\mathbb{I P}\left\{\tau_{A} \leq 2 \Delta \bigcap \mathcal{N}_{2}^{0}\right\}
$$

Podemos majorar o fator da direita na somatória (3.19) por

$$
I P\left\{\bigcap_{i=0}^{k-j-2} \mathcal{N}_{1}^{i} \bigcap\left(\tau_{A} \circ T^{(k-j-1) f_{A}} \leq 2 \Delta\right) \bigcap \mathcal{N}_{2}^{k-j-1}\right\}
$$

e similarmente o fator da direita na somatória (3.20) por

$$
\alpha(2 \Delta-n) \mathbb{P}\left\{\bigcap_{i=1}^{k-j-1} \mathcal{N}_{1}^{i}\right\} \leq \alpha(\Delta) \mathbb{I P}\left\{\bigcap_{i=1}^{k-j-1} \mathcal{N}_{1}^{i}\right\} .
$$

Finalmente, usamos a propriedade misturadora para obter a majoração 


$$
\begin{aligned}
\mathbb{P}\left\{\bigcap_{i=0}^{\ell} \mathcal{N}_{1}^{i} \cap B\right\} & \leq \mathbb{P}\left\{\bigcap_{i=0}^{\ell-1} \mathcal{N}_{1}^{i} \cap B\right\} \\
& \leq \prod_{i=1}^{\ell-1}\left[\mathbb{P}\left\{\mathcal{N}_{1}^{i}\right\}+\alpha(\Delta)\right][\mathbb{P}\{B\}+\alpha(\Delta)] \\
& =\left[\mathbb{P}\left\{\mathcal{N}_{1}^{0}\right\}+\alpha(\Delta)\right]^{\ell-1}[\mathbb{P}\{B\}+\alpha(\Delta)]
\end{aligned}
$$

para qualquer evento $B \in \mathcal{F}_{\left\{(\ell+1) f_{A},(\ell+2) f_{A}+n-1\right\}}$. Agora escolhemos $\Delta$ tal que

$$
\alpha(\Delta)=\mathbb{P}\left\{\tau_{A} \circ T^{\Delta} \leq \Delta \bigcap \tau_{A} \circ T^{2 \Delta}>f_{A}-2 \Delta\right\}
$$

\section{Portanto}

$$
\begin{aligned}
\mathbb{P}\{\mathcal{N}\} & =\mathbb{P}\left\{\tau_{A} \circ T^{2 \Delta}>f_{A}-2 \Delta\right\} \\
& =\mathbb{P}\left\{\tau_{A} \circ T^{\Delta}>f_{A}-\Delta\right\}+\mathbb{P}\left\{\tau_{A} \circ T^{\Delta} \leq \Delta \bigcap \tau_{A} \circ T^{2 \Delta}>f_{A}-2 \Delta\right\} \\
& =\mathbb{P}\left\{\mathcal{N}_{1}^{0}\right\}+\alpha(\Delta) .
\end{aligned}
$$

Assim, obtemos que o parâmetro da geométrica na equação (3.24) é $\mathbb{P}\{\mathcal{N}\}$.

Aplicando a desigualdade (3.24) à equação (3.22) com $B=\left\{\tau_{A} \circ T^{(k-j-1) f_{A}} \leq 2 \Delta \cap \mathcal{N}_{2}^{k-j-1}\right\}$, e à equação (3.23) com $B=\Omega$ e somando, obtemos a majoração (3.17).

Mais ainda, por definição, $\Delta$ é tal que $\alpha(\Delta) \leq \Delta I P\{A\}$. Portanto, se $\Delta_{1}$ é o que realiza o $\inf _{x}[x \mathbb{P}\{A\}+\alpha(x)]$, temos que $\Delta \geq \Delta_{1}$, com o que $\alpha(\Delta) \leq \alpha\left(\Delta_{1}\right)$. Por outro lado,

$$
\begin{aligned}
\mathbb{P}\{B\}+\alpha(\Delta) & =\mathbb{P}\left\{\tau_{A} \leq 2 \Delta \bigcap \mathcal{N}_{2}^{0}\right\}+\alpha(\Delta) \\
& =\mathbb{P}\left\{\tau_{A} \leq \Delta \bigcap \mathcal{N}_{1}^{0}\right\}+\mathbb{P}\left\{\tau_{A} \circ T^{\Delta} \leq \Delta \bigcap \mathcal{N}_{2}^{0}\right\}+\alpha(\Delta) \\
& \leq 3 \alpha(\Delta)
\end{aligned}
$$


Isto conclui a prova da condição sobre $\Delta$.

Para provar a segunda desigualdade, temos pelo Teorema do Valor Médio

$$
\left|\mathbb{P}\left\{\tau_{A}>f_{A}-2 \Delta\right\}^{k}-\mathbb{P}\left\{\tau_{A}>f_{A}\right\}^{k}\right| \leq k 2 \Delta \mathbb{P}\{A\} \mathbb{P}\left\{\tau_{A}>f_{A}-2 \Delta\right\}^{k-1}
$$

Isto conclui a prova da proposição.

Estamos em condições agora de provar o Teorema 3.1.

\subsubsection{Prova do Teorema 3.1.}

Definamos primeiramente

$$
\widetilde{\lambda_{A}}=\frac{-\log \mathbb{I P}\left\{\tau_{A}>f_{A}-2 \Delta\right\}}{f_{A} I P\{A\}}
$$

com $\Delta$ e $f_{A}$ tais que $n<\Delta<f_{A} / 3$. Notemos que

$$
\widetilde{\lambda_{A}}=\lambda_{A, f-2 \Delta} \frac{\left(f_{A}-2 \Delta\right) I P\{A\}}{f_{A} I P\{A\}} .
$$

Portanto, $\widetilde{\lambda_{A}} \in\left[2 \Lambda_{1} / 3, \Lambda_{2}\right]$, onde $\Lambda_{1}, \Lambda_{2}$, são os mesmos do Lema 3.9. Desta maneira, redefinimos a partir de agora, este intervalo como o dado pelo Teorema 3.1.

Para $t=k f_{A}+r$, com $k$ e $r$ inteiros, $0 \leq r<f_{A}$, pomos

$$
\begin{aligned}
\left|\mathbb{P}\left\{\tau_{A}>t\right\}-e^{-\widetilde{\lambda_{A}} P\{A\} t}\right| & \leq\left|\mathbb{P}\left\{\tau_{A}>k f_{A}+r\right\}-\mathbb{P}\left\{\tau_{A}>k f_{A}\right\}\right| \\
& +\left|\mathbb{P}\left\{\tau_{A}>k f_{A}\right\}-\mathbb{P}\{\mathcal{N}\}^{k}\right| \\
& +\left|e^{-\widetilde{\lambda_{A}} P\{A\} k f_{A}}-e^{-\widetilde{\lambda_{A}} P\{A\} t}\right| .
\end{aligned}
$$


O primeiro termo é controlado usando a equação (3.7) e a propriedade misturadora

$$
\begin{aligned}
\left|\mathbb{P}\left\{\tau_{A}>k f_{A}+r\right\}-\mathbb{P}\left\{\tau_{A}>k f_{A}\right\}\right| & =\left|\mathbb{P}\left\{\tau_{A}>k f_{A}+r\right\}-\mathbb{P}\left\{\tau_{A} \circ T^{r}>k f_{A}\right\}\right| \\
& =\mathbb{P}\left\{\tau_{A} \leq r \bigcap \tau_{A} \circ T^{r}>k f_{A}\right\} \\
& \leq r \mathbb{P}\{A\}\left[\mathbb{P}\left\{\mathcal{N}_{1}^{1}\right\}+\alpha(\Delta)\right]^{k-1} \\
& \leq C f_{A} \mathbb{P}\{A\} e^{-\widetilde{\lambda_{A}} \boldsymbol{P}\{A\} k f_{A}} \\
& \leq C f_{A} \mathbb{P}\{A\} e^{-\widetilde{\lambda_{A}} \boldsymbol{P}\{A\} t}
\end{aligned}
$$

onde $\Delta$ é o escolhido segundo a equação (3.25).

Uma majoração similar é obtida para o terceiro termo usando o Teorema do Valor Meio

$$
\begin{aligned}
\left|e^{-\widetilde{\lambda_{A}} P\{A\} k f_{A}}-e^{-\widetilde{\lambda_{A}} P\{A\} t}\right| & \leq \widetilde{\lambda_{A}} \mathbb{P}\{A\} r e^{-\widetilde{\lambda_{A}} P\{A\} k f_{A}} \\
& \leq C \Lambda_{2} \mathbb{P}\{A\} f_{A} e^{-\widetilde{\lambda_{A}} P\{A\} t} .
\end{aligned}
$$

Finalmente, o segundo termo é majorado pela propriedade da independência

$$
\begin{aligned}
\left|\mathbb{P}\left\{\tau_{A}>k f_{A}\right\}-\mathbb{P}\{\mathcal{N}\}^{k}\right| & \leq C \Delta \mathbb{P}\{A\} k \mathbb{P}\{\mathcal{N}\}^{k-2} \leq C \frac{\Delta \mathbb{P}\{A\}}{f_{A}} t e^{-\widetilde{\lambda_{A}} P\{A\} k f_{A}} \\
& \leq C \frac{\Delta \mathbb{P}\{A\}}{f_{A}} t e^{-\widetilde{\lambda_{A}} \boldsymbol{P}\{A\} t} .
\end{aligned}
$$

A soma destas três majorações dá o controle para todo $t$.

Para provar a parte 1 do teorema escolhemos $f_{A}$ tal que 


$$
\frac{\Delta}{f_{A}}=f_{A} \mathbb{I P}\{A\}
$$

Para a parte 2 do teorema definimos $\delta_{A}=\sup _{f_{A}}\left\{\widetilde{\lambda_{A, f}}\right\}$.

Isto, junto com o Lema 3.9 conclui a prova do teorema.

Lema 3.12 Seja $\mu$ um inteiro positivo. Existe um $n_{0}$ tal que para todo $n \geq n_{0}$, existem constantes positivas $\Upsilon_{1}=\Upsilon_{1}(\mu), \Upsilon_{2}=\Upsilon_{2}(\mu)$ tais que

$$
0<\Upsilon_{1}(\mu)<\mathbb{P}\left\{\tau_{A}>\mu \frac{1}{\mathbb{P}\{A\}}\right\}<\Upsilon_{2}(\mu)<1
$$

para todo n-cilindro $A$.

Prova. Por uma aplicação direta do Teorema 3.1 e do Lema 3.4

$$
\begin{aligned}
\mathbb{P}\left\{\tau_{A}>\mu \frac{1}{\operatorname{P}\{A\}}\right\} & \leq e^{-\dot{\xi}_{A} P\{A\} \mu \frac{1}{P\{A\}}}+\mathbb{P}\{A\}^{\beta} \leq e^{-\Lambda_{1} \mu}+e^{-c n} \\
& :=\Lambda_{2}(\mu)
\end{aligned}
$$

que é menor que 1 para $n$ suficentemente grande.

Uma conta similar prova a outra desigualdade.

Isto conclui a prova do lema.

Observação A condição (3.12) é usada no Lema 3.9 para provar a desigualdade direita de (3.14). O valor $1 / 2$ nesta condição é simbólico e pode ser reemplazado por qualquer valor menor que 1. Uma vez provado o Teorema 3.1, usamo-lo para estimar o valor de $\mathbb{I P}\left\{\tau_{A} \leq \mu \frac{1}{\boldsymbol{P}\{A\}}\right\}$ para qualquer valor $\mu>0$ fixo. Este valor resulta estritamente menor que 1. Portanto, agora vale o Lema 3.9 sobre a condição $f_{A} I P\{A\} \leq \mu$, e temos que $\Lambda_{2}=\Lambda_{2}(\mu)$ (re-definindo os dados pelo Teorema 3.1). Assumimos então, de agora em diante, esta condição. Notar assim mesmo que, para a desigualdade esquerda do Lema 3.9 , realmente só é necesaria a condição de que $f_{A} I P\{A\}$ seja limitado superiormente (por alguma constante). 


\subsubsection{Prova do Teorema 3.2.}

Seja $t=k \frac{1}{\boldsymbol{P}\{A\}}+r$, com $k$ inteiro não negativo e com $0<r<\frac{1}{\boldsymbol{P}\{A\}}$. Suponhamos primeiramente que $r=\frac{p}{q} \frac{1}{\boldsymbol{P}\{A\}}$, com $p / q$ fração irredutível. Suponhamos também que $q \leq \frac{1}{\widetilde{C} \Delta \boldsymbol{P}\{A\}}$, onde $\widetilde{C}$ é uma constante que escolheremos a posteriori. Denotamos $\xi_{A}=\widetilde{\lambda_{A, f}} \operatorname{com} f_{A}=\frac{1}{\boldsymbol{P}\{A\}}$.

$$
\begin{aligned}
& \left|\mathbb{P}\left\{\tau_{A}>t\right\}-e^{-\xi_{A} P\{A\} t}\right| \\
= & \left|\mathbb{P}\left\{\tau_{A}>k \frac{1}{\mathbb{P}\{A\}}+r\right\}-\mathbb{P}\left\{\tau_{A}>\frac{1}{\mathbb{P}\{A\}}-2 \Delta\right\}^{k+P\{A\} r}\right| \\
\leq & \left|\mathbb{P}\left\{\tau_{A}>k \frac{1}{\mathbb{P}\{A\}}+r\right\}-\mathbb{P}\left\{\tau_{A}>k \frac{1}{\mathbb{P}\{A\}}\right\} \mathbb{P}\left\{\tau_{A}>r\right\}\right| \\
+ & \left|\mathbb{P}\left\{\tau_{A}>k \frac{1}{\mathbb{P}\{A\}}\right\} \mathbb{P}\left\{\tau_{A}>r\right\}-\mathbb{P}\left\{\tau_{A}>\frac{1}{\mathbb{P}\{A\}}-2 \Delta\right\}^{k} \mathbb{P}\left\{\tau_{A}>r\right\}\right| \\
+ & \left|\mathbb{P}\left\{\tau_{A}>\frac{1}{\mathbb{P}\{A\}}-2 \Delta\right\}^{k} \mathbb{P}\left\{\tau_{A}>r\right\}-\mathbb{P}\left\{\tau_{A}>\frac{1}{\mathbb{P}\{A\}}-2 \Delta\right\}^{k+P\{A\} r}\right| .
\end{aligned}
$$

Se $k=0$, os dois primeiros termos na majoração acima estão ausentes. Se $k \geq 1$, o primeiro termo à direita da desigualdade é controlado usando a demostração do Lema (3.10) com $u=$ $(k-1) \frac{1}{P\{A\}}$, e a equação $(3.24)$

$$
\begin{aligned}
& \left|\mathbb{P}\left\{\tau_{A}>k \frac{1}{\mathbb{P}\{A\}}+r\right\}-\mathbb{P}\left\{\tau_{A}>k \frac{1}{\mathbb{P}\{A\}}\right\} \mathbb{P}\left\{\tau_{A}>r\right\}\right| \\
\leq & C \Delta \mathbb{P}\{A\} \mathbb{P}\left\{\tau_{A}>(k-1) \frac{1}{\mathbb{P}\{A\}}\right\} \\
\leq & C \Delta \mathbb{P}\{A\} \mathbb{P}\left\{\tau_{A}>\frac{1}{\mathbb{P}\{A\}}-2 \Delta\right\}^{k-1} \\
\leq & C \Delta \mathbb{P}\{A\} e^{-\xi_{A} P\{A\} t} .
\end{aligned}
$$

O segundo termo é controlado pela equação (3.18) 


$$
\begin{aligned}
& \left|\mathbb{P}\left\{\tau_{A}>k \frac{1}{\mathbb{P}\{A\}}\right\} \mathbb{P}\left\{\tau_{A}>r\right\}-\mathbb{P}\left\{\tau_{A}>\frac{1}{\mathbb{P}\{A\}}-2 \Delta\right\}^{k} \mathbb{P}\left\{\tau_{A}>r\right\}\right| \\
\leq & \mathbb{P}\left\{\tau_{A}>r\right\} C \Delta \mathbb{P}\{A\} k \mathbb{P}\left\{\tau_{A}>\frac{1}{\mathbb{P}\{A\}}-2 \Delta\right\}^{k} \\
\leq & C \Delta \mathbb{P}\{A\}^{2} t e^{-\xi_{A} P\{A\} t} .
\end{aligned}
$$

Veremos como controlar o terceiro termo.

Este fator é limitado superiormente usando o Teorema do Valor Médio aplicado à função $h(x)=x^{1 / q}$ e considerando que $h(x)$ é convexa por baixo.

$$
\begin{aligned}
& \left|\mathbb{P}\left\{\tau_{A}>\frac{1}{\mathbb{P}\{A\}}-2 \Delta\right\}^{k} \mathbb{P}\left\{\tau_{A}>r\right\}-\mathbb{P}\left\{\tau_{A}>\frac{1}{\mathbb{P}\{A\}}-2 \Delta\right\}^{k+P\{A\} r}\right| \\
= & \mathbb{P}\left\{\tau_{A}>\frac{1}{\mathbb{P}\{A\}}-2 \Delta\right\}^{k}\left|\mathbb{P}\left\{\tau_{A}>r\right\}-\mathbb{P}\left\{\tau_{A}>\frac{1}{\mathbb{P}\{A\}}-2 \Delta\right\}^{P\{A\} r}\right| .
\end{aligned}
$$

O fator da direita é controlado da seguinte maneira

$$
\begin{aligned}
& \left|\mathbb{P}\left\{\tau_{A}>r\right\}-\mathbb{P}\left\{\tau_{A}>\frac{1}{\mathbb{P}\{A\}}-2 \Delta\right\}^{P\{A\} r}\right| \\
= & \left|\mathbb{P}\left\{\tau_{A}>\frac{1}{\mathbb{P}\{A\}} \frac{p}{q}\right\}^{q}-\mathbb{P}\left\{\tau_{A}>\frac{1}{\mathbb{P}\{A\}}-2 \Delta\right\}^{p}\right| \times \frac{1}{q} \omega^{\frac{1}{q}-1} \\
\leq & \left|\mathbb{P}\left\{\tau_{A}>\frac{1}{\mathbb{P}\{A\}} \frac{p}{q}\right\}^{q}-\mathbb{P}\left\{\tau_{A}>\frac{1}{\mathbb{P}\{A\}}-2 \Delta\right\}^{p}\right| \times \\
& \times \frac{1}{q} \min \left\{\mathbb{P}\left\{\tau_{A}>\frac{1}{\mathbb{P}\{A\}} \frac{p}{q}\right\}^{q} ; \mathbb{P}\left\{\tau_{A}>\frac{1}{\mathbb{P}\{A\}}-2 \Delta\right\}^{p}\right\}^{\frac{1}{q}}-1 \\
= & \frac{|b-a|}{a} \frac{1}{q} a^{\frac{1}{q}},
\end{aligned}
$$

onde chamamos de $b$ e $a$ ao máximo e mínimo respeitivamente da diferença (3.32) e onde $\omega$ é tal que $a<\omega<b$. Temos que 


$$
\frac{|b-a|}{a} \leq \frac{|b-c|}{a}+\frac{|c-a|}{a}
$$

para qualquer $c$, real positivo. Escolhemos $c=\mathbb{P}\left\{\tau_{A}>\frac{1}{P\{A\}} p\right\}$.

Consideraremos agora, dois casos. i) Quando $a$ é o membro da direita e ii) quando $a$ é o membro da esquerda da diferença (3.32).

Prova do caso $i$ ).

Pela equação (3.17) com $k=p$ e $f_{A}=\frac{1}{P\{A\}}$

$$
\frac{|c-a|}{a} \frac{1}{q} \leq C \frac{\Delta \mathbb{P}\{A\} p a}{a} \frac{1}{\mathbb{I P}\left\{\tau_{A}>\frac{1}{P\{A\}}\right\}^{2}} \frac{1}{q} \leq C \Delta \mathbb{P}\{A\} .
$$

Pela equação (3.18) com $k=q$ e $f_{A}=\frac{1}{\boldsymbol{P}\{A\}}$

$$
\begin{aligned}
\frac{|b-c|}{a} \frac{1}{q} & \leq C \frac{\Delta \mathbb{P}\{A\} q b}{a} \frac{1}{\mathbb{P}\left\{\tau_{A}>\frac{1}{P\{A\}}\right\}^{2}} \frac{1}{q} \leq C \frac{\Delta \mathbb{P}\{A\}}{a} \frac{c}{1-C^{\prime} \Delta \mathbb{P}\{A\} q} \\
& \leq C \frac{\Delta \mathbb{P}\{A\}}{a} \frac{1+C \Delta \mathbb{P}\{A\} p}{1-C^{\prime} \Delta \mathbb{P}\{A\} q} a \leq C \Delta \mathbb{P}\{A\}
\end{aligned}
$$

onde a última desigualdade é satisfeita se escolhemos $\widetilde{C}>C^{\prime}$.

Prova do caso ii).

Novamente, pela equação (3.18)

$$
\frac{|a-c|}{a} \frac{1}{q} \leq C \frac{\Delta \mathbb{P}\{A\} q a}{a} \frac{1}{q} \leq C \Delta \mathbb{P}\{A\} .
$$


Similarmente, pela equação (3.17)

$$
\begin{aligned}
\frac{|c-b|}{a} \frac{1}{q} & \leq C \frac{\Delta \mathbb{P}\{A\} p b}{a} \frac{1}{q} \leq C \frac{\Delta \mathbb{P}\{A\}}{a} \frac{c}{1-C^{\prime \prime} \Delta \mathbb{P}\{A\} p} \\
& \leq C \frac{\Delta \mathbb{P}\{A\}}{a} \frac{1+C \Delta \mathbb{P}\{A\} q}{1-C^{\prime \prime} \Delta \mathbb{P}\{A\} q} a \leq C \Delta \mathbb{P}\{A\}
\end{aligned}
$$

onde a última desigualdade é satisfeita se escolhemos $\widetilde{C}>C^{\prime \prime}$.

Escolhemos finalmente $\widetilde{C}=\max \left\{C^{\prime} ; C^{\prime \prime} ; 2\right\}+1$. Isto prova que o terceiro termo é limitado superiormente por

$$
C \Delta \mathbb{P}\{A\} \mathbb{P}\left\{\tau_{A}>\frac{1}{\mathbb{P}\{A\}}-2 \Delta\right\}^{k} \leq C \Delta \mathbb{P}\{A\} e^{-\xi_{A} P\{A\} t}
$$

Seja agora $t$ qualquer. Escrevemos $t=k \frac{1}{\boldsymbol{P}\{A\}}+r$, com $k$ e $r$ inteiros, $0 \leq r<\frac{1}{\boldsymbol{P}\{A\}}$. Podemos escolher um $\bar{t}$ tal que $\bar{t}<t$ e $\bar{t}=k \frac{1}{\boldsymbol{P}\{A\}}+\underset{q}{p} \operatorname{com} p / q$ fração irredutível e $|t-\bar{t}|<\widetilde{C} \Delta \mathbb{P}\{A\}$.

$$
\begin{aligned}
\left|\mathbb{P}\left\{\tau_{A}>t\right\}-e^{-\dot{\xi}_{A} \boldsymbol{P}\{A\} t}\right| & \leq\left|\mathbb{P}\left\{\tau_{A}>t\right\}-\mathbb{P}\left\{\tau_{A}>\bar{t}\right\}\right| \\
& +\left|\mathbb{P}\left\{\tau_{A}>\bar{t}\right\}-e^{-\xi_{A} P\{A\} \bar{t}}\right| \\
& +\left|e^{-\xi_{A} \boldsymbol{P}\{A\} \bar{t}}-e^{-\xi_{A} \boldsymbol{P}\{A\} t}\right|
\end{aligned}
$$

Posto que $\mathbb{P}\left\{\tau_{A}>t\right\}$ é constante em cada intervalo $[k, k+1), k$ inteiro, temos que

$$
\left|\mathbb{P}\left\{\tau_{A}>t\right\}-\mathbb{P}\left\{\tau_{A}>\bar{t}\right\}\right|=0
$$


A majoração para o terceiro termo é obtida usando o Teorema do Valor Meio

$$
\begin{aligned}
\left|e^{-\xi_{A} P\{A\} \bar{t}}-e^{-\xi_{A} P\{A\} t}\right| & \leq \xi_{A} \mathbb{I P}\{A\}\left(r-\frac{p}{q}\right) e^{-\xi_{A} P\{A\} \bar{t}} \\
& \leq C \Lambda_{2} \Delta \mathbb{P}\{A\} e^{-\xi_{A} P\{A\} t} .
\end{aligned}
$$

Finalmente, o segundo termo é majorado como na primeira parte da demostração.

Isto, junto ao Lema 3.12 com $\mu=1$ e à equação (3.26), conclui a prova do teorema.

\subsubsection{Aplicação: Estimação de $\mathbb{E}\left(\tau_{A}\right)$}

Um resultado importante na teoria ergódica e o famoso Lema de Kac que disse que a esperança do tempo de volta a um evento raro é exatamente o inverso da medida do evento.

Gostariamos de ter um resultado semelhante para a esperança do tempo de entrada em um evento raro. Desafortunadamente tal resultado não existe e é poco o que se conhece ao respeito do valor de tal esperança (ver por exemplo, Saussol (1998)).

Apresentaremos no próximo resultado uma estimação do valor da esperança do tempo de entrada. Se a lei do tempo de entrada converge para uma lei exponencial, esta lei deverá ter parâmetro $\xi_{A}=1 / \mathbb{E}\left(\tau_{A}\right)$, pois na lei exponencial o parâmetro é o inverso da esperança e claramente esperamos que ambas as leis possuam a mesma esperança.

A estimação da lei do tempo de entrada apresssentada no Teorema 3.2 em função do tempo $t$ e não somente uma majoração uniforme em termos da medida do evento $A$ é fundamental para demostrar uma tal estimação.

Corolário 3.13 Nas condições do Teorema 3.1 vale a seguinte desigualdade

$$
\left|\mathbb{E}\left(\xi_{A} \mathbb{P}\{A\} \tau_{A}\right)-1\right| \leq C \Delta \mathbb{P}\{A\}
$$

ou eequivalentemente,

$$
\left|\frac{\mathbb{E}\left(\tau_{A}\right)}{\mathbb{E}_{A}\left(\tau_{A}\right)}-\frac{1}{\xi_{A}}\right| \leq C \Delta \mathbb{P}\{A\}
$$


onde $\Delta$ é o mesmo do Teorema 3.2.

Em particular, a equação (3.33) geraliza o limite no ponto 3 do Teorema provado em Galves e Schmitt (1990) para processos $\phi$-misturador com decaimento exponencial.

Prova. Primeiro, notamos que aplicando a mudança de variáveis $s=\xi_{A} I P\{A\} t$, no Teorema 3.2 , temos que

$$
\left|\mathbb{P}\left\{\tau_{A}>\frac{s}{\xi_{A} \mathbb{P}\{A\}}\right\}-e^{-s}\right| \leq C \Delta \mathbb{P}\{A\} s e^{-s}+C \Delta \mathbb{P}\{A\} e^{-s}
$$

Usando agora o Teorema do Valor Médio

$$
e^{-(1-\Delta \boldsymbol{P}\{A\}) s}-e^{-s}=\Delta \mathbb{P}\{A\} s e^{-\left(1-\chi_{A}\right) s} \geq \Delta \mathbb{P}\{A\} s e^{-s} .
$$

$\operatorname{com} \chi_{A} \in(1-\Delta \mathbb{P}\{A\}, 1)$. Deste modo

$$
\begin{aligned}
\left|\mathbb{E}\left(\xi_{A} \mathbb{I P}\{A\} \tau_{A}\right)-1\right| & \leq C[\mathbb{E}(\exp \{1-\Delta \mathbb{P}\{A\}\})-\mathbb{E}(\exp \{1\})+\Delta \mathbb{P}\{A\} \mathbb{E}(\exp \{1\})] \\
& =C\left[\frac{1}{1-\Delta \mathbb{P}\{A\}}-1+\Delta \mathbb{P}\{A\}\right] \leq C \Delta \mathbb{P}\{A\}
\end{aligned}
$$

onde denotamos com $\exp \{\nu\}$ uma variável aleatória exponencial de parâmetro $\nu$.

Isto conclui a prova.

\subsection{Minoração uniforme}

Uma primeira minoração surge do fato de estarmos aproximando uma distribuição discreta por uma contínua. 
Teorema 3.14 Seja $\left\{X_{m}\right\}_{m \in \mathbb{Z}}$ um processo estocástico $\phi$-misturador qualquer ou $\alpha$-misturador com a somável. Então para qualquer $n$ e qualquer $A \in \mathcal{C}_{n}$, vale a seguinte desigualdade

$$
C_{1} \mathbb{P}\{A\} \leq \sup _{t>0}\left|\mathbb{P}\left\{\tau_{A}>t\right\}-e^{-\xi_{A} P\{A\} t}\right|
$$

Prova. A prova segue a prova da Proposição 2.3 em Hirata-Saussol-Vaienti, 1998.

Posto que $\mathbb{P}\left\{\tau_{A}>t\right\}$ é constante em $[[t],[t]+1)=[k, k+1)$, temos que para todo $0<\delta<1$

$$
\begin{aligned}
\sup _{t \in[k, k+\delta)}\left|\mathbb{P}\left\{\tau_{A}>t\right\}-e^{-\xi_{A} \boldsymbol{P}\{A\} t}\right| & \geq \frac{1}{2}\left|e^{-\xi_{A} \boldsymbol{P}\{A\} k}-e^{-\xi_{A} \boldsymbol{P}\{A\}(k+\delta)}\right| \\
& \geq e^{-\xi_{A} \boldsymbol{P}\{A\} k} \frac{1-e^{-\Lambda_{1} \boldsymbol{P}\{A\} \delta}}{2} \\
& \geq C \Lambda_{1} \mathbb{P}\{A\} \delta e^{-\Lambda_{2} \boldsymbol{P}\{A\} k} .
\end{aligned}
$$

Em particular

$$
\sup _{t>0}\left|\mathbb{P}\left\{\tau_{A}>t\right\}-e^{-\xi_{A} P\{A\} t}\right| \geq C_{1} \mathbb{P}\{A\}
$$

Uma consequência do Teorema 3.2 foi uma majoração uniforme para o erro na aproximação exponencial do instante de entrada. O objetivo do teorema anterior é apresentar uma minoração uniforme. Desta forma, o fato de termos pedido no teorema anterior que o processo seja misturador, obedece ao fato de apresentá-lo como a contra-parte do anterior resultado e portanto, re-escalando a lei de entrada no evento raro pelo parâmetro $\lambda_{A}$.

Não obstante, devemos notar que para a aproximação à lei exponencial de parâmetro $\xi_{A}=1$ o teorema é muito mais geral.

Corolário 3.15 Seja $\left\{X_{m}\right\}_{m \in \mathbb{Z}}$ um processo estocástico estacionário. Escrevamos $A=A_{n}(\omega)$. A seguinte desigualdade é verdadeira

$$
C_{1} \mathbb{P}\{A\} \leq \sup _{t>0}\left|\mathbb{P}\left\{\tau_{A}>t\right\}-e^{-P\{A\} t}\right|
$$

Prova. A prova é idêntica à anterior. 


\subsection{Comportamento do parâmetro de re-escalamento}

Nós provamos que a lei do instante de ocorrência de um evento raro pode ser bem aproximada por uma lei exponencial de parâmetro $\xi_{A} I P\{A\}$ onde $\xi_{A}$ é limitado por duas constantes positivas. No entanto, para um evento fixo $A \in \mathcal{C}_{n}$, esse valor depende em geral de uma quantidade de coordenadas do processo muito maior que $n$ (lembrar definição de $\xi_{A}$ ). Nas próximas seções, apresentaremos uma estimação do valor do parâmetro $\xi_{A}$ assim como o seu comportamento típico.

\subsubsection{Estimação do parâmetro de re-escalamento}

Recordamos brevemente que $\lambda_{A, f}$ foi definido em (3.13).

Seja $s$ um inteiro positivo. Lembramos que $\mathbb{P}_{A}$ denota a medida de probabilidade condicionada em $A$ e definimos

$$
\zeta_{A, s}=\mathbb{P}_{A}\left\{\tau_{A}>\frac{n}{s}\right\}=\frac{\mathbb{P}\left\{\left(\tau_{A}>\frac{n}{s}\right) \cap A\right\}}{\operatorname{IP}\{A\}}
$$

Teorema 3.16 Seja $\left\{X_{m}\right\}_{m \in \mathbb{Z}}$ um processo exponencialmente $\alpha$-misturador ou $\phi$-misturador qualquer. Seja s um inteiro positivo. Então, existem constantes estritamente positivas $\Psi_{1}, \Psi_{2}, C_{1}$, $C_{2}$ e c tais que para quaisquer $n$ e $A \in \mathcal{C}_{n}, \zeta_{A, s} \in\left[\Psi_{1}, \Psi_{2}\right]$, e também vale a seguinte desigualdade

$$
C_{1} \mathbb{P}\{A\} \leq \sup _{t>0}\left|\mathbb{P}\left\{\tau_{A}>\frac{t}{\zeta_{A, s} \mathbb{P}\{A\}}\right\}-e^{-t}\right| \leq C_{2} e^{-c n} .
$$

Prova. Para um inteiro positivo $k \leq f_{A}$, definamos $\sigma_{A, k}=\mathbb{P}\left\{\tau_{A} \leq k\right\} / k \mathbb{P}\{A\}$. Então

$$
\begin{aligned}
\left|e^{-\lambda_{A, f} \boldsymbol{P}\{A\} t}-e^{-\zeta_{A, s} P\{A\} t}\right| & \leq\left|e^{-\lambda_{A, f} \boldsymbol{P}\{A\} t}-e^{-\sigma_{A, f} P\{A\} t}\right| \\
& +\left|e^{-\sigma_{A, f} P\{A\} t}-e^{-\zeta_{A, s} P\{A\} t}\right|
\end{aligned}
$$


Primeiro notemos que $\theta \leq 1-e^{-\theta}+\theta^{2} / 2$, para todo $\theta \in[0,1]$. Então, escolhendo $\theta=$ $-\log \mathbb{P}\left\{\tau_{A}>k\right\}$,

$$
\sigma_{A, k} \leq \frac{-\log \mathbb{P}\left\{\tau_{A}>k\right\}}{k \mathbb{P}\{A\}} \leq \sigma_{A, k}+2 k \mathbb{P}\{A\}
$$

para todo $k \leq f_{A}$ e portanto

$$
\left|\sigma_{A, k}-\lambda_{A, k}\right| \leq C k I P\{A\}
$$

O segundo termo é majorado decompondo-o nos somandos com $j \leq n / s$ e $j>n / s$, e usando as propriedades de mistura nos somandos do segundo termo.

Para o caso $\alpha$-misturador temos que,

$$
\begin{aligned}
& \left|e^{-\sigma_{A, f} \boldsymbol{P}\{A\} t}-e^{-\zeta_{A, s} \boldsymbol{P}\{A\} t}\right| \\
\leq & \left|\sigma_{A, f}-\zeta_{A, s}\right| \\
& =\frac{1}{f_{A} \mathbb{P}\{A\}}\left|\sum_{j=1}^{f_{A}}\left[\mathbb{P}\left\{\tau_{A}=n / s\right\}-\mathbb{P}\left\{\tau_{A}=j\right\}\right]\right| \\
\leq & \frac{1}{f_{A} \mathbb{P}\{A\}}\left[\sum_{j=n / s}^{f_{A}} \mathbb{P}\left\{\tau_{A} \leq j-\frac{n}{s} \cap \tau_{A} \circ T^{j-\frac{n}{s}}=\frac{n}{s}\right\}+2 \frac{n}{s} \mathbb{P}\{A\}\right] \\
\leq & \left.\frac{1}{f_{A} \mathbb{P}\{A\}} \sum_{j=n / s}^{f_{A}} \mathbb{P}\left\{\tau_{A} \leq j-\frac{n}{s} \cap T^{-j} A^{\left(\frac{n}{2 s}\right)}\right)\right\}+2 \frac{n}{s f_{A}} \\
\leq & \frac{1}{f_{A} \mathbb{P}\{A\}} \sum_{j=n / s}^{f_{A}}\left[\mathbb{P}\left\{\tau_{A} \leq j-\frac{n}{s}\right\} \mathbb{P}\left\{A^{\left(\frac{n}{2 s}\right)}\right\}+\mathbb{P}\left\{\tau_{A} \leq j-\frac{n}{s}\right\} \alpha\left(\frac{n}{2 s}\right)\right] \\
+ & 2 \frac{n}{s f_{A}} \\
\leq & f_{A} \mathbb{P}\left\{A^{\left(\frac{n}{2 s}\right)}\right\}+f_{A} \alpha\left(\frac{n}{2 s}\right)+2 \frac{n}{s f_{A}} \\
\leq & C e^{-c n},
\end{aligned}
$$


onde a última desigualdade segue da eleição

$$
f_{A}=\min \left\{\frac{1}{\sqrt{\mathbb{P}\left\{A^{\left(\frac{n}{2 s}\right)}\right\}}} ; \frac{1}{\alpha\left(\frac{n}{4 s}\right)}\right\},
$$

e do Lema 3.4.

Para o caso $\phi$-misturador a conta é a mesma atê o termo (3.35). Neste caso, podemos majorar esta expressão por

$$
\begin{aligned}
& \leq \frac{1}{f_{A} \mathbb{P}\{A\}} \sum_{j=n / s}^{f_{A}}\left[\mathbb{P}\left\{\tau_{A} \leq j-\frac{n}{s}\right\} \mathbb{P}\left\{A^{\left(\frac{n}{2 s}\right)}\right\}+\mathbb{P}\left\{\tau_{A} \leq j-\frac{n}{s}\right\} \mathbb{P}\left\{A^{\left(\frac{n}{2 s}\right)}\right\} \phi\left(\frac{n}{2 s}\right)\right] \\
& +2 \frac{n}{s f_{A}} \\
& \leq f_{A} \mathbb{P}\left\{A^{\left(\frac{n}{2 s}\right)}\right\}+f_{A} \mathbb{P}\left\{A^{\left(\frac{n}{2 s}\right)}\right\} \phi\left(\frac{n}{2 s}\right)+2 \frac{n}{s f_{A}} \\
& \leq C e^{-c n}
\end{aligned}
$$

onde a última desigualdade segue da eleição $f_{A}=1 / \sqrt{\mathbb{I P}\left\{A^{\left(\frac{n}{2 s}\right)}\right\}}$.

A majoração do teorema segue da primeira parte do Teorema 3.1.

Mais ainda, por definição $\zeta_{A, s} \leq 1=\Psi_{2}$. Posto que $\left|\sigma_{A, f}-\zeta_{A, s}\right| \leq C e^{-c n}$ e $\left|\sigma_{A, f}-\lambda_{A, f}\right| \leq$ $C f_{A} \mathbb{I P}\{A\}$, se sigue que existe $\Psi_{1} \leq \zeta_{A, s}$.

Isto conclui a prova.

Observação No Teorema 3.2 provamos que $\xi_{A} \in\left[\Lambda_{1}, \Lambda_{2}\right] \ni 1$. Teorema 3.16 estabelece que $\zeta_{A, s} \in\left[\Psi_{1}, 1\right]$. Podemos deducir que, na verdade, $\xi_{A} \in\left[\Lambda_{1}, 1+C_{2} e^{-c n}\right]$, onde $C_{2}$ e $c$ são as mesmas constantes que aparecem em (3.34). Isto, junto com o Corolario 3.13, prova o que já é conhecido como folclore e foi comentado em Shields (1996), isto é, que em geral, instantes de ocorrência são maiores que instantes de retorno. 


\subsubsection{Limite quase-certo}

Generalizaremos agora a definição 2.2 .

Definição 3.17 Seja $s \in \mathbb{N}$. Definimos $\mathcal{B}_{n}=\mathcal{B}_{n}(s)$ como o conjunto dos $A \in \mathcal{C}_{n}$ que recorrem antes do instante $n / s$, isto é, $A \in \mathcal{B}_{n}$, se e somente, se existe um inteiro $1 \leq k \leq[n / s]-1$ tal que $A \cap T^{-k} A \neq \emptyset$.

No próximo lema, provaremos que a classe de cilindros para os quais o $\zeta_{A, s}=1$, é típica no seguinte sentido

Lema 3.18 Seja $\left\{X_{m}\right\}_{m \in \mathbb{Z}}$ um processo $\alpha$-misturador (decaindo a qualquer velocidade). Existe $s \in \mathbb{N}$ e duas constantes positivas $C$ e c tais que

$$
\mathbb{I P}\left\{\mathcal{B}_{n}(s)\right\} \leq C e^{-c n}
$$

Prova. Seja $s$ um inteiro positivo. Tomemos $A=\left\{X_{1}^{n}=a_{1}^{n}\right\}$. Primeiro observamos que existe um $k$, com $1 \leq k \leq[n / s]-1$, tal que

$$
a_{k}^{n}=a_{1}^{n-k+1}
$$

o que implica

$$
a_{j k+1}^{(j+1) k}=a_{1}^{k}
$$

para todo $0 \leq j \leq[n / k]-1$. Seja $m(k)=[n / k]$, então 


$$
\begin{aligned}
& \leq \sum_{a_{1}^{n} \in \mathcal{B}_{n}(s)} \mathbb{P}\left\{\mathcal{B}_{n}(s)\right\} \\
& \leq \sum_{k=2}^{n / s} \sum_{b_{1}^{k} \in \mathcal{E}^{k}} \mathbb{P}\left\{X_{1}^{n}=a_{1}^{n}\right\} \\
& \leq \sum_{k=2}^{n / s} \sum_{b_{1}^{k} \in \mathcal{E}^{k}}\left(\alpha(0)+\mathbb{P}\left\{X_{1}^{k}=b_{1}^{k}\right\}\right) \mathbb{P}\left\{X_{k+1}^{2 k}=\cdots=X_{(m(k)-1) k+1}^{m(k) k}=b_{1}^{k}, X_{m(k) k+1}^{m(k) k+r}=b_{1}^{r}\right\} \\
& \left.\leq \sum_{k=2}^{n / s} \sum_{b_{1}^{k} \in \mathcal{E}^{k}}\left(\alpha(0)+\mathbb{P}\left\{X_{1}^{k}=b_{1}^{k}\right\}\right) C e^{-c n(s-1) / s}=b_{1}^{k}\right\} \\
& \leq C \alpha(0) e^{n\left[\frac{\ln |\mathcal{E}|-c(s-1)}{s}\right]}+C e^{-c n},
\end{aligned}
$$

onde usamos a propriedade $\alpha$-misturadora e Lema 3.4 junto com o fato de ser $n-k \geq n-n / s$. Tomando $s$ suficientemente grande obtemos o decaimento exponencial.

Isto conclui a prova do lema.

Na linguagem de sistemas dinâmicos, em geral é escolhido um ponto genérico $\omega \in \Omega$ e a sequência de cilindros $A_{n}=\left\{X_{0}^{n-1}=\omega_{0}^{n-1}\right\}$ cujas medidas convergem para zero em torno a esse ponto $\omega$.

O Lema 3.18 nos permite mostrar o seguinte resultado

Corolário 3.19 Assumamos que o processo $\left\{X_{m}\right\}_{m \in \mathbb{Z}}$ é estacionário e exponencialmente $\alpha$ misturador. Existem constantes positivas $c, C_{1}$ e $C_{2}$ tal que a seguinte desigualdade é válida para quase todo $\omega \in \Omega$

$$
C_{1} \mathbb{I P}\left\{A_{n}(\omega)\right\} \leq \sup _{t \geq 0}\left|\mathbb{P}\left\{\tau_{A_{n}(\omega)}>\frac{t}{\mathbb{P}\left\{A_{n}(\omega)\right\}}\right\}-e^{-t}\right| \leq C_{2} e^{-c n}
$$

Prova. Se $A \in \mathcal{B}_{n}$, então $\zeta_{A, s}=1$. O resto é consequência do Lema 3.18 e do Teorema 3.16 . 
Exemplo 3.20 Proporcionamos agora um exemplo de um processo e uma sequência de cilindros $A_{n}$ tais que $\xi_{A_{n}}<C<1$ para todo n natural e para alguma constante $C$.

Sejam $\mathcal{E}=\{0,1\}$ e $A=A_{n}=\left\{X_{0}=1, \ldots, X_{n-1}=1\right\}$ para todo inteiro positivo $n$. Suponhamos que $X_{n}$ são variáveis aleatorias independentes identicamente distribuídas com $\mathbb{P}\left\{X_{n}=1\right\}=p$. Então $\zeta_{A, s}=1-p$.

\subsection{Critério para lei não degenerada}

Nós provamos no Lema 3.9 que a obtenção de uma minoração uniforme $\Lambda_{1}$ para o parâmetro $\xi_{A}$ depende do controle do segundo momento da função de contagem $N$ das ocorrências do evento $A$ até o instante $f_{A}$. Essa condição foi suficiente para a minoração uniforme. Embora na prática, essa condição pareça ser a mais adequada para controlar, apresentaremos na próxima proposição uma condição que mostra que a dita hipótese não é necessáaria. Mais ainda, apresentamos uma condição necessária e suficiente que, informalmente, diz que o momento que deve ser controlado é o momento de "ordem logarítmico mais um".

Proposição 3.21 Seja $\left\{X_{m}\right\}_{m \in \mathbb{Z}}$ um processo estocástico estacionário. Seja $t>0$. Denote$\operatorname{mos} N=N_{[t]}$. Então

$$
\mathbb{P}\left\{\tau_{A} \leq t\right\}=e^{-\boldsymbol{E}\left(\frac{N}{t P\{A\}} \log \frac{N}{t P\{A\}}\right)}
$$

onde adotamos a convenção $(N \log N)(0)=0$.

Prova. Pela desigualdade de Hölder com $p$ e $q$ conjugados temos

$$
\mathbb{E} N=\mathbb{E}\left(N \mathbb{1}_{\{\operatorname{sopp} N\}}\right) \leq \mathbb{E}\left(N^{p}\right)^{1 / p} \mathbb{P}\{N \geq 1\}^{1 / q}
$$

e a igualdade vale no limite quando $p$ tende a 1 .

Agora, posto que por definição $\mathbb{E} N=t \mathbb{P}\{A\}$, por um cálculo direto temos

$$
\mathbb{P}\left\{\tau_{A} \leq t\right\}=\mathbb{P}\{\mathbb{I N} \geq 1\}=\lim _{p \rightarrow 1} \frac{1}{\left(\mathbb{E}\left(\frac{N}{t P\{A\}}\right)^{p}\right)^{1 /(p-1)}}=\frac{1}{e^{E\left(\frac{N}{t P\{A\}} \log \frac{N}{t P\{A\}}\right)}}
$$


Isto conclui a prova da proposição.

Corolário 3.22 Nas condições do Teorema 3.1, existe $\Lambda_{1}>0$ tal que $\Lambda_{1} \leq \xi_{\text {A }}$ para todo $A \in \mathcal{C}_{n}$, se e somente se, existe uma constante positiva $C$ tal que $\mathbb{E}\left(N_{\frac{1}{P\{A\}}} \log N_{\frac{1}{P\{A\}}}\right) \leq C$. Mais ainda, neste caso,

$$
\Lambda_{1}=\inf _{A} \exp \left\{-\mathbb{E}\left(\mathbb{N} \frac{1}{P\{A\}} \log \mathbb{I N} \frac{1}{P\{A\}}\right)\right\}
$$

Prova. A equação (3.14) da a equivalência.

O proprio Lema 3.9 e a proposição anterior concluem a prova do corolário.

\subsection{Processos uniformemente misturadores}

Os processos uniformemente misturadores são uma família muito ampla de processos. Como já vimos, a não hipótese sobre o decaimento da função $\gamma$ equivale à condição (1.2). Ante tal generalidade não é possível obter a convergência para a lei exponencial do instante de entrada, embora, quando o decaimento da função $\gamma$ é suficientemente rápido e o cilindro é não recorrente ainda se pode obter a convergência uniforme para esta lei.

Mesmo que a técnica usada aqui seja similar à usada para processos $\alpha$-misturadores e $\phi$-misturadores apresentamos o resultado de maneira separada para obseervar o fato que a majoração aqui obtida é uniforme e não em função de $t$ como nos casos anteriores e portanto, também não vale a estimação de $\mathbb{E}\left(\tau_{A}\right)$ para este caso.

Ainda que os processos uniformemente misturadores gerais não possuam a propriedade de decaimento exponencial uniforme dos cilindros (pois, se for o caso, todo sistema deste tipo possuiria entropia positiva, o qual não é certo segundo veremos mais adiante) um decaimento uniforme, dependendo do decaimento da função $\gamma$, pode ser provado.

Lema 3.23 Seja $\left\{X_{m}\right\}_{m \in \mathbb{Z}}$ um processo $\gamma$-misturador. Existem constantes estritamente positivas $C$ e $\Gamma$ tais que para todo inteiro positivo $n$ e para todo cilindro $A \in \mathcal{C}_{n}$ vale a seguinte desigualdade

$$
\mathbb{P}\{A\} \leq C\left[e^{-\Gamma \sqrt[3]{n}}+\gamma(\sqrt[3]{n})\right]
$$


Prova. Escrevamos $A=\left\{X_{1}^{n}=a_{1}^{n}\right\}$. Notemos que $n=[\sqrt{n}]^{2}+r$, com $0 \leq r \leq 2 n$.

Observamos primeiro que

$$
\mathbb{P}\left\{X_{1}^{n}=a_{1}^{n}\right\} \leq \mathbb{P}\left\{X_{1}=a_{1}, X_{[\sqrt{n}]+1}=a_{[\sqrt{n}]+1}, X_{2[\sqrt{n}]+1}=a_{2[\sqrt{n}]+1}, \ldots\right\}
$$

Usando a propriedade misturadora temos

$$
\mathbb{I P}\{A\} \leq \rho^{[\sqrt{n}]}+\gamma([\sqrt{n}]-1) \frac{1-\rho^{[\sqrt{n}]}}{1-\rho}
$$

onde $\rho=\sup \left\{\mathbb{P}\left\{a_{i}\right\}: a_{i} \in \mathcal{E}\right\}$, e por hipótese, $\rho<1$. Agora, só resta notar que, $\sqrt[3]{n} \leq[\sqrt{n}]-1$ para todo $n>n_{0}$.

Isto conclui a prova do lema.

Observação Os processos uniformemente misturadores gerais não possuem a propriedade de decaimento exponencial uniforme. Como já vimos, esta propriedade implica que o processo possui entropia positiva. Esta última condição é equivalente ao processo possuir a propriedade de $K$-mistura. Por outro lado, existem processos $K$-misturadores, não uniformemente misturadores (ver definição, propriedades e Teorema 1, §8, Cap. 10 em Cornfeld, Fomin e Sinai (1982)).

Teorema 3.24 Seja $\left\{X_{m}\right\}_{m \in \mathbb{Z}}$ um processo estocástico estacionário uniformemente misturador com função $\gamma$. Seja s um inteiro positivo. Existem duas constantes $0<\Lambda_{1}<\Lambda_{2}<\infty$, tais que para todos os $A \in \mathcal{E}^{n} \backslash \mathcal{B}_{n}(s)$, com $\sum_{j \geq n / s} \gamma(j) \leq C \mathbb{P}\{A\}$, existe uma constante positiva $\widetilde{\lambda_{A}} \in\left[\Lambda_{1}, \Lambda_{2}\right]$, tal que as seguintes desigualdades são verdadeiras

$$
C \mathbb{P}\{A\} \leq \sup _{t \geq 0}\left|\mathbb{P}\left\{\tau_{A}>t\right\}-e^{-\widetilde{\lambda_{A}} P\{A\} t}\right| \leq C \mathbb{P}\{A\}^{\beta} .
$$


Prova. Definimos primeiramente $\widetilde{\lambda_{A}}$ como na equação (3.26). Seguindo a prova da Proposição 3.11 , neste caso podemos majorar a somatória (3.19) por

$$
\sum_{j=0}^{k-2} \mathbb{P}\{\mathcal{N}\}^{j} \Delta \mathbb{P}\{A\} \leq \frac{1}{\mathbb{P}\left\{\tau_{A} \leq f_{A}-2 \Delta\right\}} \Delta \mathbb{P}\{A\}
$$

Similarmente majoramos a somatória (3.20) por

$$
\sum_{j=0}^{k-2} \mathbb{P}\{\mathcal{N}\}^{j} \gamma(\Delta) \leq \frac{1}{\mathbb{P}\left\{\tau_{A} \leq f_{A}-2 \Delta\right\}} \gamma(\Delta)
$$

Finalmente, o termo (3.21) por

$$
\mathbb{P}\{\mathcal{N}\}^{k-1} \Delta \mathbb{P}\{A\}
$$

O denominador nas expressões acima é controlado usando a equação (3.10) e usando a prova da minoração de $\widetilde{\lambda_{A}}$.

A majoração de $\widetilde{\lambda_{A}}$ se segue como no Lema 3.9 .

Para a minoração devemos controlar os termos presentes no lado direito da equação (3.11).

$$
\begin{aligned}
\mathbb{E}\left(N^{2}\right)=\sum_{l=1}^{[t]} \mathbb{E}\left(\mathbb{1}_{T^{-l}(A)}\right) & +2 \sum_{l=1}^{n / s}([t]-l) \mathbb{E}\left(\mathbb{1}_{A} \mathbb{1}_{T^{-l}(A)}\right) \\
& +2 \sum_{l=n / s+1}^{2 n}([t]-l) \mathbb{E}\left(\mathbb{1}_{A} \mathbb{1}_{T^{-l}(A)}\right) \\
& +2 \sum_{l=2 n+1}^{t}([t]-l) \mathbb{E}\left(\mathbb{I}_{A} \mathbb{1}_{T^{-l}(A)}\right) .
\end{aligned}
$$


Já é claro que o primeiro termo na decomposição acima é $t \mathbb{I P}\{A\}$ e o segundo é zero. As somatórias nos dois termos restantes são controlados pela mistura uniforme.

O terceiro termo é majorado por

$$
\sum_{l=n / s+1}^{2 n}([t]-l)\left[\mathbb{P}\{A\} \mathbb{P}\left\{A^{(n / 2 s)}\right)+\gamma(l-n / 2 s)\right] \leq t \mathbb{P}\{A\} n \mathbb{P}\left\{A^{(n / 2 s)}\right\}+t \sum_{j=n / 2 s}^{2 n-n / 2 s} \gamma(j) .
$$

Finalmente, o último termo é majorado por

$$
\sum_{l=2 n+1}^{t}([t]-l)\left[\mathbb{P}\{A\}^{2}+\gamma(l-n)\right] \leq \frac{t^{2} \mathbb{I P}\{A\}^{2}}{2}+t \sum_{j=n+1}^{t-n} \gamma(j)
$$

De maneira que, pelo argumento do Lema 3.9, devemos provar que os termos da direita das equações (3.36) e (3.37) são de ordem menor o igual a $t \mathbb{P}\{A\}$. Pelo Lema $3.23, n \mathbb{P}\left(A^{(n / 2 s)}\right) \rightarrow$ 0 . É imediato concluir que existe $0<\Lambda_{1}<\xi_{A}$, para todo $A \in \mathcal{C}_{n}$ e para todo $n$ se existe uma constante $C$, tal que

$$
\frac{\sum_{j=n / 2 s}^{t} \gamma(j)}{\mathbb{P}\{A\}} \leq C<\infty
$$

Escolhemos agora $t=f_{A}$. Escolhemos $\Delta$ como no Teorema 3.2 e finalmente $f_{A}$ tal que $\Delta / f_{A}=f_{A} \mathbb{P}\{A\}$.

Isto conclui a prova do teorema. 


\section{Capítulo 4}

\section{INSTANTE DE VOLTA E TEMPO DE PERMANÊNCIA}

\subsection{Motivação}

No capítulo anterior, vimos qual é, aproximadamente, a lei de entrada num evento raro. Isto é, qual é a distribuição do tempo que leva até observar um evento $A$ com probabilidade $\mathbb{I P}\{A\} \ll 1$ observando o processo desde o equilíbrio.

A pergunta que nos motiva agora é: Qual é, aproximadamente, a lei de retorno ou volta a um evento raro. Ou seja, agora estamos interessados em descrever o tempo que leva até observar o evento $A$, dado que o processo está em $A$.

Esta questão é fundamental para entender posteriormente o processo pontual das sucessivas passagens pelo conjunto $A$ que chamaremos de instantes de ocorrência do evento $A$. Observe-se que ao considerar a primeira entrada em $A$, estamos considerando o tempo de entrada, enquanto, as subseqüentes entradas em $A$ correspondem a tempos de retorno no evento $A$.

Provaremos então, qual é o limite assintótico para a lei de volta. Veremos que este não é uma lei exponencial, mas sim, uma combinação de uma lei exponencial com uma medida de Dirac concentrada em um ponto que depende das propriedades locais de recorrência inerentes ao evento. O resultado valerá para uma família ampla de processos misturadores. Provaremos também a velocidade de convergência para dita lei. 


\subsection{Instante de volta}

Nesta secão aplicamos os resultados sobre tempo de entrada para estudar o tempo de volta. Veremos que o tempo de volta a um evento $A$ depende das propriedades de recorrência desse evento. A próxima definição será necessária para definir tais propriedades.

Definição 4.1 Para $A \in \mathcal{C}_{n}$ definimos a ordem de recorrência de A (respeito do operador $T$ ) da seguinte maneira

$$
r_{A}=\inf \left\{1 \leq x \in \mathbb{I} \mid A \cap T^{-x}(A) \neq \emptyset\right\} .
$$

Definição 4.2 Denotamos com $\mathcal{R}_{r}=\mathcal{R}_{r}(n)$, a familia de cilindros de ordem de recorrência $r$, isto é

$$
\mathcal{R}_{r}=\left\{A \in \mathcal{C}_{n} \mid r_{A}=r\right\}
$$

Notamos que $\mathcal{R}_{r}$ com $1 \leq r \leq n$, formam uma partição de $\mathcal{C}_{n}$. Tomemos $A \in \mathcal{R}_{r}$ e escrevamos $n=q r+s$, com $0 \leq s<r$. Temos então

$$
A=\left\{X_{0}^{r-1}=X_{r}^{2 r-1}=\ldots=X_{(q-1) r}^{q r-1}=a_{1}^{r}, X_{q r}^{n-1}=a_{1}^{s}\right\}
$$

Assim, diremos que $A$ possui período $r$ e resto $s$. Notar que neste caso, os períodos também podem ser lidos "de frente para trais", ou seja

$$
A=\left\{X_{n-r}^{n-1}=X_{n-2 r}^{n-1-r}=\ldots X_{n-q r}^{n-(q-1) r-1}=a_{n-r}^{n-1}, X_{0}^{s-1}=a_{n-s}^{n-1}\right\}
$$

Seja $1 \leq j \leq n$. Notamos que $A \cap T^{-j}(A) \neq \emptyset$, se e somente se, $j=k r$ com $1 \leq k \leq q$.

Denotemos com $G=\left\{X_{n}^{n+r-1} \neq a_{s}^{s+r-1}\right\}$. Assim, se $A$ é um $n$-cilindro de período $r$ e resto $s$, então $\mathbb{P}_{A}\{G\}=\mathbb{P}_{A}\left\{\tau_{A} \neq r\right\}=\mathbb{P}_{A}\left\{\tau_{A}>r\right\}$. 
Queremos provar que a lei do tempo de volta a um evento raro converge assintoticamente a uma mistura de uma lei de Dirac e uma lei exponencial. Definamos $\mathcal{L}(t)$ da seguinte maneira

$$
\mathcal{L}(t)= \begin{cases}1 & t<r \\ \mathbb{P}_{A}\{G\} & r \leq t \leq n \\ \mathbb{P}_{A}\{G\} e^{-\xi_{A} P\{A\} t} & t>n\end{cases}
$$

onde $\xi_{A}$ é o mesmo do Teorema 3.2.

O seguinte lema será usado na prova do próximo teorema.

Lema 4.3 Seja $\left\{X_{m}\right\}_{m \in \mathbb{Z}}$ um processo $\alpha$-misturador qualquer. Seja $A \in \mathcal{C}_{n}$ e $M \in \mathbb{I N}$. Então, a seguinte desigualdade é verdadeira

$$
\mathbb{P P}_{A}\left\{\tau_{A} \circ T^{n} \leq M\right\} \leq \inf _{1 \leq w \leq n} n\left[\mathbb{P}\left\{A^{(w)}\right\}+\alpha(n-w)\right]+M[\mathbb{P}\{A\}+\alpha(n)]
$$

Prova. Consideramos $M>n$. Em caso contrário o segundo termo na majoração desaparece. A majoração resulta a mesma nos dois casos. Seja $1 \leq w \leq n$. A propriedade de mistura implica que

$$
\begin{aligned}
& \mathbb{P}\left\{A \cap \tau_{A} \circ T^{n} \leq M\right\} \\
= & \sum_{j=0}^{n-1} \mathbb{P}\left\{A \cap \tau_{A} \circ T^{n}=j\right\}+\sum_{j=n}^{M} \mathbb{P}\left\{A \cap \tau_{A} \circ T^{n}=j\right\} \\
\leq & \sum_{j=0}^{n-1} \mathbb{P}\left\{A \cap T^{-(n+j)}\left(A^{(w)}\right)\right\}+\sum_{j=n}^{M} \mathbb{P}\left\{A \cap T^{-(n+j)}(A)\right\} \\
\leq & \sum_{j=0}^{n-1} \mathbb{P}\{A\} \mathbb{P}\left\{T^{-(n+j)}\left(A^{(w)}\right)\right\}+\sum_{j=0}^{n-1} \mathbb{P}\{A\} \alpha(n+j-w) \\
+ & \sum_{j=n}^{M} \mathbb{P}\{A\} \mathbb{P}\left\{T^{-(n+j)}(A)\right\}+\sum_{j=n}^{M} \mathbb{P}\{A\} \alpha(j),
\end{aligned}
$$

logo, usando a estacionaridade da medida temos que 


$$
\mathbb{P}_{A}\left\{A \cap \tau_{A} \circ T^{n} \leq M\right\} \leq n\left[\mathbb{P}\left\{A^{(w)}\right\}+\alpha(n-w)\right]+M[\mathbb{P}\{A\}+\alpha(n)]
$$

Isto conclui a prova posto que $w$ é arbitrário.

Teorema 4.4 Seja $\left\{X_{m}\right\}_{m \in \mathbb{Z}}$ um processo $\alpha$-misturador com $\alpha$ somável. Então, existem constantes positivas $C_{1}$ e $C_{2}$ tais que para qualquer $n$ e qualquer $A \in \mathcal{C}_{n}$, a seguinte desigualdade é verdadeira

$$
\begin{aligned}
\left|\mathbb{P}_{A}\left\{\tau_{A}>t\right\}-\mathcal{L}(t)\right| & \leq C_{1} \inf _{2 n \leq \Delta \leq \frac{1}{P\{A\}}}[\Delta[\mathbb{P}\{A\}+\alpha(n)]+\alpha(\Delta-2 n)] \\
& +C_{2} \inf _{1 \leq w \leq n} n\left[\mathbb{P}\left\{A^{(w)}\right\}+\alpha(n-w)\right] .
\end{aligned}
$$

Prova Para $t \leq n$, posto que $r$ é a ordem de recorrência de $A$ temos

$$
\mathbb{P}_{A}\left\{\tau_{A}>t\right\}=\mathcal{L}(t)
$$

Agora, escolhemos $\Delta$ como em (4.1). Para $t \geq \Delta$ temos

$$
\begin{aligned}
\left|\mathbb{P}_{A}\left\{\tau_{A}>t\right\}-\mathcal{L}(t)\right| & \leq\left|\mathbb{P}_{A}\left\{\tau_{A}>t\right\}-\mathbb{P}_{A}\left\{G \cap \tau_{A} \circ T^{\Delta}>t-\Delta\right\}\right| \\
& +\left|\mathbb{P}_{A}\left\{G \cap \tau_{A} \circ T^{\Delta}>t-\Delta\right\}-\mathbb{P}_{A}\{G\} \mathbb{P}\left\{\tau_{A} \circ T^{\Delta}>t-\Delta\right\}\right| \\
& +\mathbb{P}_{A}\{G\}\left|\mathbb{P}\left\{\tau_{A} \circ T^{\Delta}>t-\Delta\right\}-\mathbb{P}\left\{\tau_{A}>t\right\}\right| \\
& +\mathbb{P}_{A}\{G\}\left|\mathbb{P}\left\{\tau_{A}>t\right\}-e^{-\xi_{A} P\{A\} t}\right| .
\end{aligned}
$$

O primeiro termo no lado direito da equação acima é controlado da seguinte maneira 


$$
\begin{aligned}
& \left|\mathbb{P}_{A}\left\{\tau_{A}>t\right\}-\mathbb{P}_{A}\left\{G \cap \tau_{A} \circ T^{\Delta}>t-\Delta\right\}\right| \\
= & \left|\mathbb{P}_{A}\left\{G \cap \tau_{A} \circ T^{n}>t-n\right\}-\mathbb{P}_{A}\left\{G \cap \tau_{A} \circ T^{\Delta}>t-\Delta\right\}\right| \\
\leq & \mathbb{P}_{A}\left\{G \cap \tau_{A} \circ T^{n} \leq \Delta-n\right\} \\
\leq & \mathbb{P}_{A}\left\{\tau_{A} \circ T^{n} \leq \Delta-n\right\} .
\end{aligned}
$$

E este último termo é controlado usando o Lema 4.3

$$
\begin{aligned}
\mathbb{P}_{A}\left\{\tau_{A} \circ T^{n} \leq \Delta-n\right\} & \leq \inf _{1 \leq w \leq n} n\left[\mathbb{P}\left\{A^{(w)}\right\}+\alpha(n-w)\right] \\
& +\Delta[\mathbb{I P}\{A\}+\alpha(n)] .
\end{aligned}
$$

O segundo termo é majorado usando a propriedade $\alpha$-misturadora

$$
\begin{aligned}
& \left|\mathbb{P}_{A}\left\{G \cap \tau_{A} \circ T^{\Delta}>t-\Delta\right\}-\mathbb{P}_{A}\{G\} \mathbb{P}\left\{\tau_{A} \circ T^{\Delta}>t-\Delta\right\}\right| \\
\leq & \alpha(\Delta-(n+r)) \leq \alpha(\Delta-2 n) .
\end{aligned}
$$

Para o terceiro termo temos

$$
\mathbb{P}_{A}\{G\}\left|\mathbb{P}\left\{\tau_{A} \circ T^{\Delta}>t-\Delta\right\}-\mathbb{P}\left\{\tau_{A}>t\right\}\right| \leq \Delta \mathbb{P}\{A\}
$$

Para o último termo usamos o Teorema 3.2

$$
\begin{aligned}
\mathbb{P}_{A}\{G\} \mid \mathbb{P}\left\{\tau_{A}>t\right\}-e^{-\xi_{A} P\{A\}(t-n) \mid} & \leq\left|\mathbb{P}\left\{\tau_{A}>t\right\}-e^{-\xi_{A} P\{A\} t}\right| \\
& \leq \inf _{n \leq \Delta \leq \frac{1}{P\{A\}}}[\Delta \mathbb{P}\{A\}+\alpha(\Delta)] .
\end{aligned}
$$


Para $n \leq t \leq \Delta$ temos

$$
\begin{aligned}
& \left|\mathbb{P}_{A}\left\{\tau_{A}>t\right\}-\mathcal{L}(t)\right| \\
\leq & \left|\mathbb{P}_{A}\left\{G \cap \tau_{A} \circ T^{n}>t-n\right\}-\mathbb{P}_{A}\{G\}\right|+\left|\mathbb{P}_{A}\{G\}-\mathbb{P}_{A}\{G\} e^{-\xi_{A} P\{A\} t}\right| \\
\leq & \mathbb{P}_{A}\left\{G \cap \tau_{A} \circ T^{n} \leq t-n\right\}+\mathbb{P}_{A}\{G\}\left(1-e^{-\xi_{A} P\{A\} t}\right) \\
\leq & \mathbb{P}_{A}\left\{G \cap \tau_{A} \circ T^{n} \leq \Delta-n\right\}+\left(1-e^{-\xi_{A} P\{A\} \Delta}\right) \\
\leq & \mathbb{P}_{A}\left\{\tau_{A} \circ T^{n} \leq \Delta-n\right\}+\Lambda_{2} \mathbb{P}\{A\} \Delta .
\end{aligned}
$$

E o primeiro termo é majorado usando novamente o Lema 4.3

$$
\begin{aligned}
\mathbb{P}_{A}\left\{\tau_{A} \circ T^{n} \leq \Delta-n\right\} & \leq \inf _{1 \leq w \leq n} n\left[\mathbb{P P}\left\{A^{(w)}\right\}+\alpha(n-w)\right] \\
& +\Delta[\mathbb{P}\{A\}+\alpha(n)]
\end{aligned}
$$

Isto conclui a prova do teorema.

Corolário 4.5 Seja $\left\{X_{m}\right\}_{m \in \mathbb{Z}}$ um processo $\alpha$-misturador com função $\alpha$ somável. As seguintes condições são equivalentes,

(a)

$$
\left|P_{A}\left\{\tau_{A}>t\right\}-e^{-\xi_{A} P\{A\} t}\right| \leq C e(A)
$$

(b)

$$
\left|\mathbb{P}_{A}\left\{\tau_{A}>t\right\}-\mathbb{P}\left\{\tau_{A}>t\right\}\right| \leq C e(A)
$$


(c)

$$
\left|\mathbb{P}_{A}\{G\}-1\right| \leq C e(A),
$$

onde $e(A) \geq e_{2}(A)$, e onde denotaremos $e_{1}(A)$ o erro dado pelo Teorema 3.2 e $e_{2}(A)$ o erro dado pelo Teorema 4.4. Em particular, a lei de retorno a um evento $A$ converge a uma lei exponencial se e somente se a lei de retorno e a lei de entrada convergem a uma mesma lei e se e somente se o evento $A$ é não recorrente.

Prova. $(a) \Rightarrow(b)$

$$
\begin{aligned}
\left|\mathbb{P}_{A}\left\{\tau_{A}>t\right\}-\mathbb{P}\left\{\tau_{A}>t\right\}\right| & \leq\left|\mathbb{P}_{A}\left\{\tau_{A}>t\right\}-e^{-\xi_{A} P\{A\} t}\right|+\left|e^{-\xi_{A} P\{A\} t}-\mathbb{P}\left\{\tau_{A}>t\right\}\right| \\
& \leq e(A)+e_{1}(A) .
\end{aligned}
$$

(b) $\Rightarrow(a)$

$$
\begin{aligned}
\left|\mathbb{P}_{A}\left\{\tau_{A}>t\right\}-e^{-\xi_{A} \boldsymbol{P}\{A\} t}\right| & \leq\left|P_{A}\left\{\tau_{A}>t\right\}-\mathbb{P}\left\{\tau_{A}>t\right\}\right|+\left|\mathbb{P}\left\{\tau_{A}>t\right\}-e^{-\xi_{A} \boldsymbol{P}\{A\} t}\right| \\
& \leq e(A)+e_{1}(A) .
\end{aligned}
$$

$(c) \Rightarrow(a)$ Pela desigualdade triangular e o Teorema 4.4 temos

$$
\begin{aligned}
\left|\mathbb{P}_{A}\left\{\tau_{A}>t\right\}-\mathbb{P}\left\{\tau_{A}>t\right\}\right| & \leq\left|\mathbb{P}_{A}\left\{\tau_{A}>t\right\}-\mathcal{L}(t)\right|+\left|\mathcal{L}(t)-e^{-\xi_{A} P\{A\} t}\right| \\
& \leq e_{2}(A)+\left|\mathcal{L}(t)-e^{-\xi_{A} P\{A\} t}\right|
\end{aligned}
$$

Agora 


$$
\left|\mathcal{L}(t)-e^{-\xi_{A} \boldsymbol{P}\{A\} t}\right|=\left\{\begin{array}{ll}
1-e^{-\xi_{A} \boldsymbol{P}\{A\} t} & \text { para } 0<t<r \\
\left|\mathbb{P}_{A}\{G\}-e^{-\xi_{A} \boldsymbol{P}\{A\} t}\right| & \text { para } r \leq t<n \\
\left|\mathbb{P}_{A}\{G\}-1\right| e^{-\xi_{A} \boldsymbol{P}\{A\} t} & \text { para } n \leq t
\end{array} .\right.
$$

É claro que para $0 \leq t<r$

$$
\left|1-e^{-\xi_{A} P\{A\} t}\right| \leq \Lambda_{2} \mathbb{P}\{A\} t \leq \Lambda_{2} \mathbb{P}\{A\} n
$$

Por outro lado, para $r \leq t<n$,

$$
\begin{aligned}
\left|\mathbb{P}_{A}\{G\}-e^{-\xi_{A} P\{A\} t}\right| & \leq\left|\mathbb{P}_{A}\{G\}-1\right|+\left|1-e^{-\xi_{A} P\{A\} t}\right| \\
& \leq\left|\mathbb{P}_{A}\{G\}-1\right|+\Lambda_{2} \mathbb{P}\{A\} n \\
& \leq e(A)+C \mathbb{P}\{A\} n .
\end{aligned}
$$

E para $t \geq n$,

$$
\sup _{t}\left|\mathbb{P}_{A}\{G\}-1\right| e^{-\xi_{A} P\{A\} t} \leq\left|\mathbb{P}_{A}\{G\}-1\right| \leq e(A) .
$$

$(a) \Rightarrow(c)$

$$
\left|\mathcal{L}(t)-e^{-\xi_{A} P\{A\} t}\right| \leq\left|\mathcal{L}(t)-\mathbb{P}_{A}\left\{\tau_{A}>t\right\}\right|+\left|\mathbb{P}_{A}\left\{\tau_{A}>t\right\}-e^{-\xi_{A} P\{A\} t}\right| \leq e_{2}(A)+e(A)
$$

Em particular, para $t=r$,

$$
\left|\mathbb{P}_{A}\{G\}-e^{-\xi_{A} P\{A\} r}\right| \leq e(A)+e_{2}(A),
$$




$$
\left|\mathbb{P}_{A}\{G\}-1\right| \leq\left|\mathbb{P}_{A}\{G\}-e^{-\xi_{A} \boldsymbol{P}\{A\} r}\right|+\left|e^{-\xi_{A} P\{A\} r}-1\right| \leq e(A)+\Lambda_{2} \mathbb{P}\{A\} n
$$

Isto conclui a prova do corolário.

\subsection{Tempo de permanência}

Nós provamos, na seção anterior, que o tempo de volta a um evento raro, dado que estamos no evento raro, é uma mistura de duas leis. Uma sendo uma $\delta_{r}$, isto é uma delta de Dirac, medida concentrada no ponto de recorrência do evento, com uma lei exponencial. Em poucas palavras, dado que estamos no evento $A$, ou o evento volta logo segundo a recorrência própria do evento (e neste caso o processo se repetirá); ou devemos esperar um novo tempo exponencial para observar a ocorrência do evento.

No primeiro caso, onde o evento se repete, se formarão "nuvens" onde se pode observar a ocorrência repetida do evento até o processo conseguir sair dessa nuvem.

Queremos então estudar o comportamento dessa nuvem. No próximo teorema, provamos que essa nuvem que chamaremos tempo de permanência possui, baixo uma certa regularidade da medida, uma lei que bem pode ser aproximada por uma lei geométrica com parâmetro dependendo das propriedades de recorrência do evento.

Definição 4.6 Seja $A \in \mathcal{R}_{r}, 1 \leq r<n$. Definimos o tempo de permanência como a variável aleatória $S_{A}: A \rightarrow \mathbb{N} \cup\{\infty\}$

$$
S_{A}(\omega)=\sup \left\{s \in \mathbb{N} \mid \omega \in A \cap T^{-s r} A ; \forall j=1, \ldots, s\right\}
$$

O seguinte lema e posterior definição nos permitem definir o conceito de regularidade da medida acima mencionado. 
Lema 4.7 Seja $l_{i}$ uma sequencia de números reais tais que $0<l_{i}<1$, com $i \in \mathbb{N}$. Seja $0<l<1$ e $\left|l_{i}-l\right| \leq d_{i}$ para todo $i \in \mathbb{N}$ com decrescentes $d_{i}$. Então, existe uma constante $c$, com $0<c<1$, tal que a seguinte desigualdade vale para todo $n \in \mathbb{I N}$,

$$
\left|\prod_{i=1}^{n} l_{i}-l^{n}\right| \leq n c^{n-1} d_{1} .
$$

\section{Prova.}

$$
\begin{aligned}
\left|\prod_{i=1}^{n} l_{i}-l^{n}\right| & \leq\left|\prod_{i=1}^{n} l_{i}-\prod_{i=1}^{n-1} l_{i} l+\prod_{i=1}^{n-1} l_{i} l-\prod_{i=1}^{n-2} l_{i} l^{2}+\prod_{i=1}^{n-2} l_{i} l^{2}-\ldots-l^{n}\right| \\
& \leq \sum_{i=1}^{n}\left(\prod_{j=1}^{n-i} l_{j}\right)\left|l_{n-i+1}-l\right| l^{i-1} \leq c^{n-1} \sum_{i=1}^{n} d_{i} \\
& \leq n c^{n-1} d_{1} .
\end{aligned}
$$

Onde $c \in\left(\min \left\{l_{0}, l\right\} ; \max \left\{l_{0}, l\right\}\right)$.

Definição 4.8 Dado um evento $A \in \mathcal{R}_{r}$, definimos a sequência $r_{i}(A)$ da seguinte maneira

$$
r_{i}(A):=\mathbb{P}\left\{X_{0}^{r-1}=a_{n-r}^{n-1} \mid X_{-r}^{-1}=X_{-2 r}^{-r-1}=\ldots=X_{-(i+1) r}^{-i r-1}=a_{n-r}^{n-1}, X_{-(i+1) r-s}^{-(i+1) r-1}=a_{0}^{s-1}\right\}
$$

Isto é, se A é um n-cilindro de período $r$ e resto $s, r_{i}$ é a probabilidade do processo repetir o período de $A$ dado que aconteceram $i$ vezes o período (de comprimento $r$ ) e uma vez o resto (de comprimento s) consecutivamente.

No próximo teorema, assumimos que existe o limite dos $r_{i}$ com velocidade $\gamma_{i}$, isto é, existe um número real $\ell_{A}$ tal que

$$
\left|r_{i}-\ell_{A}\right| \leq \gamma_{i}
$$

para todo $i \geq 1$, onde $\gamma_{i}$ é uma sequência de números reais positivos convergentes para zero e onde $0<\ell_{A}<1$. 
Teorema 4.9 Seja $\left\{X_{m}\right\}_{m \in \mathbb{Z}}$ um processo estacionário. Seja $A \in \mathcal{R}_{r}$. Assumamos que o limite em (4.3) existe. Então, existe uma constante c com $0<c<1$ tal que valem as seguintes desigualdades para todo $k \in \mathbb{I N}$ e onde $q=[n / r]$,

$$
\left|\mathbb{P}_{A}\left\{S_{A}=k\right\}-\left(1-\ell_{A}\right) \ell_{A}^{k}\right| \leq 2 c^{k} \sum_{i=q}^{q+k} \gamma_{i} \leq 2(k+1) c^{k} \gamma_{q}
$$

Prova Para $k=0$ apenas notamos que $\left|r_{0}-\ell_{A}\right|=\left|1-r_{0}-1-\ell_{A}\right| \leq \gamma_{0}$. Suponhamos $k \geq 1$, temos

$$
\begin{aligned}
\mathbb{P}_{A}\left\{S_{A}=k\right\} & =\frac{\mathbb{P}\left\{A, A_{1}, \ldots A_{k}, A_{k+1}^{c}\right\}}{\mathbb{P}\{A\}} \\
& =\frac{\mathbb{P}\left\{A, A_{1}, \ldots A_{k}, A_{k+1}^{c}\right\}}{\mathbb{P}\left\{A, A_{1}, \ldots A_{k}\right\}} \frac{\mathbb{P}\left\{A, A_{1}, \ldots A_{k}\right\}}{\mathbb{P}\left\{A, A_{1}, \ldots A_{k-1}\right\}} \ldots \frac{\mathbb{P}\left\{A, A_{1}\right\}}{\mathbb{P}\{A\}}
\end{aligned}
$$

onde $A_{j}=T^{-j r} A^{(r)}$. Notemos que

$$
\mathbb{I P}\left\{A, A_{1}, \ldots, A_{k}\right\}=\mathbb{P}\left\{X_{n-s}^{n-1}=a_{1}^{s}, A_{1}, \ldots, A_{q}, A_{q+1}, \ldots, A_{q+k}\right\}
$$

e portanto

$$
\frac{\mathbb{P}\left\{A, A_{1}, \ldots A_{k}\right\}}{\mathbb{P}\left\{A, A_{1}, \ldots A_{k-1}\right\}}=r_{q+k-1}
$$

Então

$$
\mathbb{P}_{A}\left\{S_{A}=k\right\}=\left(1-r_{q+k}\right) r_{q+k-1} \ldots r_{q}=\prod_{i=q}^{q+k-1} r_{i}-\prod_{i=q}^{q+k} r_{i}
$$


e pelo Lema 4.7

$$
\begin{aligned}
\left|\mathbb{P}_{A}\left\{S_{A}=k\right\}-\left(1-\ell_{A}\right) \ell_{A}^{k}\right| & \leq\left|\prod_{i=q}^{q+k-1} r_{i}-\ell_{A}^{k}\right|+\left|\prod_{i=q}^{q+k} r_{i}-\ell_{A}^{k+1}\right| \\
& \leq 2 c^{k} \sum_{i=q}^{q+k} \gamma_{i} \leq 2(k+1) c^{k} \gamma_{q} .
\end{aligned}
$$

Isto conclui a prova do teorema.

Exemplo 4.10 A condição sobre o limite (4.3) é garantida pela continuidade do potencial para uma fonte gibbsiana e pela propriedade 2 para uma cadeia com conexões completas.

No seguinte exemplo, veremos como a lei geométrica é alcançada realmente, de maneira que essas "nuvens", onde o evento se repete, realmente existem. Ele também mostra como a parte degenerada da lei do instante de volta está presente e então, esta lei difere da lei do instante de ocorrência.

Exemplo 4.11 Seja $\left\{X_{m}\right\}_{m \in \mathbb{Z}}$ uma cadeia de Markov irredutível e aperiódica. Seja $A \in \mathcal{R}_{r}$. Então a sequencia $r_{i}$ é constante, mais precisamente e pela propriedade markoviana

$$
r_{i}=\mathbb{P}\left\{X_{n}^{n+r-1}=a_{n-r}^{n-1} \mid X_{n-1}=a_{n-1}\right\}
$$

para todo $i \in \mathbb{I N}$. Desta maneira, $\ell_{A}=r_{i}$. Mais ainda, $\mathbb{P}_{A}\{G\}=1-\ell_{A}$, e

$$
\mathbb{P}_{A}\left\{S_{A}=k\right\}=\left(1-\ell_{A}\right) \ell_{A}^{k}
$$




\section{Capítulo 5}

\section{PROCESSO DE POISSON}

\subsection{Exemplos}

A idéia geral é que se o primeiro instante de ocorrência de um evento raro possui, aproximadamente, uma lei exponencial e se considerarmos eventos raros não recorrentes então o processo pontual dos sucessivos instantes de ocorrência terá aproximadamente lei de Poisson.

Exemplo 5.1 O exemplo clássico imediato que surge quando consideramos este problema é o da convergência da lei binomial para a Poisson. Isto é, se $X_{n}$ são variáveis aleatórias i.i.d. com $\mathbb{P}\left\{X_{n}=1\right\}=p=1-\mathbb{P}\left\{X_{n}=0\right\}$ com $n \geq 1$ e se $\mathcal{N}(n)=\sum_{i=1}^{n} \mathbb{I}_{\left\{X_{n}=1\right\}}$ então

$$
\mathcal{L}(\mathcal{N}) \Rightarrow \mathcal{L}(\mathcal{Z})
$$

quando $n \rightarrow \infty$, onde $\Rightarrow$ significa convergência em distribuição e onde $Z$ é uma variável aleatória com distribuição de Poisson de parâmetro $\lambda$ sempre que $\lambda=n$ p. Ver por exemplo Feller (1976).

Exemplo 5.2 Um segundo exemplo provem da teoria de números. Para um número real $x$ qualquer definimos a parte fracionaria de $x$ como sendo o valor $\{x\}=x-[x]$. Estamos interessados na distribuição da parte fracionaria de um polinômio. Seja o polinômio de primeiro grau $p(x)=b x+a$, com b real e a irracional. Então, a sequência $\{p(n)\}$ com $n \in \mathbb{I N}$ é uniformemente distribuída no intervalo [0,1]. Mais geralmente, Weyl (1916) provou que esse fato é verdadeiro para qualquer polinômio de grau $r \geq 1$ com coeficientes reais e pelo menos um deles (exceto o coeficiente principal), irracional. O fato de, para cada n fixo, os $\{p(n)\}$ serem uniformemente distribuídos para todo $n$ equivale a o processo de occorrências dos $\{p(i)\}, i=1,2, \ldots$, formar um processo de Poisson (ver por exemplo, Lema 5.6 em Ferrari e Galves (1997)). 
Exemplo 5.3 O teorema anterior vale também no caso de funções exponenciais. Se $f(x)=$ $b a^{x}$ com $b$ real e $a>1$, então os $\{f(n)\}$ são uniformemente distribuídos e portanto, acontecem segundo um processo pontual de Poisson.

Exemplo 5.4 Döeblin (1940) estuda a aproximação poissoneana para a transformada de Gauss. Esta é definida como a função $G(x)=\{1 / x\}$ para $x \in(0,1)$. Se $x \in(0,1)$, então pode ser escrito como fração continua $x=\left[a_{1}, a_{2}, \ldots\right]$ com $a_{i}$ naturais. Mais ainda, $a_{i}=\left[1 / G^{k}(x)\right]$. Esta decomposição é finita, se e somente se, $x$ é racional. Quando $x$ é escolhido ao acaso os $a_{i}$ formam um processo estocástico. Quando $x$ é escolhido segundo a medida

$$
\mathbb{P}\{A\}=\frac{1}{\log 2} \int_{A} \frac{1}{1+x} d x
$$

para todo A mensurável Lebesgue, o processo $\left\{a_{i}\right\}_{i \in N}$ é uniformemente misturador e a medida $\mu$ é absolutamente continua respeito da medida de Lebesgue. Definimos a sequência de eventos $A_{n}$ com $n \in \mathbb{N}$ da seguinte maneira

$$
A_{n}=\left\{x \in(0,1) \mid a_{0} \geq n\right\} .
$$

Então, quando $n$ é grande, $A_{n}$ é um evento raro. Podemos considerar o processo pontual $\left\{\tau_{A_{n}}^{j}\right\}_{j \in N}$ das sucessivas passagens do processo $\left\{a_{i}\right\}_{i \in N}$ pelo conjunto $A_{n}$. Döeblin prova aconverência deste processo pontual para um processo de Poisson.

Uma análise mais detalhada dos três exemplos acima mencionados assim como a prova do Teorema de Weyl e do caso exponencial, e os fatos básicos de frações contínuas pode ser achada em Cornfeld, Fomin e Sinai (1982).

Exemplo 5.5 Collet e Coelho (1994) estudam o processo pontual dos instantes em que duas trajetórias independentes ficam a uma distáncia menor que $0<\epsilon \ll 1$ para uma transformação do círculo expansora e suave por pedaços. Eles demonstram a convergência assintótica quando $\epsilon \rightarrow 0$ para uma lei de Poisson para os instantes de sucessivas entradas numa vizinhança de tamanho $\epsilon$ e a convergência assintótica para uma lei de Poisson marcada para os instantes de sucessivas visitas a uma tal vizinhança. 


\subsection{O método Chen-Stein}

O método Chen-Stein é uma poderosa ferramenta para mostrar a convergência a uma distribuição de Poisson. Este método foi primeiramente introduzido por Stein (1972) para mostrar convergência em distribuição à normal para soma de variáveis dependentes em uma generalização do teorema de limite central.

Esta técnica é baseada na equação diferencial

$$
f^{\prime}(x)-x f(x)=h(x)-\mathbb{E} h(Z)
$$

onde Z é uma variável aleatória com distribuição normal standard e $h$ é uma função de teste. A idéia é a seguinte: se $W$ é uma variável aleatória qualquer e se define o operador

$$
\mathcal{L} f(x)=f^{\prime}(x)-x f(x)
$$

então, $\mathbb{E} \mathcal{L} f(W)=0$, para toda função diferenciável $f$ tal que $\mathbb{E}|Z f(Z)|<\infty$, se e somente, se $W$ possui distribuição normal standard. É de esperar então que se $\mathbb{E} \mathcal{L} f(W)$ é pequeno para funções $f$ então, a distribuicão de $W$ é próxima de uma distribuição normal standard.

Em 1975, Chen usou a técnica de Stein para estudar a aproximação binomial à distribuição de Poisson no caso de variáveis dependentes. Se agora $Z$ representa uma variável aleatória com distribuição de Poisson de parâmetro $\lambda$, podemos definir um operador análogo ao de (5.1) e neste caso

$$
\mathcal{L} f(x)=\lambda f(x+1)-x f(x)
$$

Desta maneira, $\mathbb{E} \mathcal{L} f(W)=0$, para toda função diferenciável $f$ tal que $\mathbb{E}|Z f(Z)|<\infty$, se e somente se, $W$ possui distribuição de Poisson de parâmetro $\lambda$. Se $W$ é uma soma de variáveis aleatórias dependentes de Bernoulli com esperança pequena, então $W$ possui distribuição aproximadamente Poisson.

Assim, o método de Chen e Stein é o primeiro que mostra esta convergência provendo também uma velocidade de convergência para processos com correlação positiva. 
Para uma análise detalhada do método, referimos o leitor a Arratia, Goldstein e Gordon (1989). Para futura referência e notação apresentaremos aqui o teorema tal e como aparece no mencionado trabalho.

Definimos primeiramente a distância em variação total entre duas distribuições. Se $X$ e $Y$ são duas variáveis aleatórias e $\mathcal{L}(X)$ e $\mathcal{L}(X)$ são suas distribuições escreveremos

$$
\begin{aligned}
\|\mathcal{L}(X)-\mathcal{L}(X)\| & =2 \sup _{A}|\mathbb{P}\{X \in A\}-\mathbb{P}\{Y \in A\}| \\
& =2 \min \mathbb{P}\{X \neq Y\}
\end{aligned}
$$

Seja $I$ um conjunto finito ou enumerável (de índices). Para cada $\alpha \in I$, seja $X_{\alpha}$ uma variável aleatória com $0<\mathbb{I P}\left\{X_{\alpha}=1\right\}=p_{\alpha}=1-\mathbb{P}\left\{X_{\alpha}=0\right\}$. Sejam $W=\sum_{\alpha \in I} X_{\alpha}$ e $0<\lambda=\mathbb{E} W<\infty$. Para cada $\alpha \in I$ definimos uma vizinhança de $\alpha$ como um conjunto $B_{\alpha} \subseteq I$ com $\alpha \in B_{\alpha}$. Seja $Z$ uma variável aleatória com $\mathbb{I E} Z=\lambda$. Definimos

$$
\begin{gathered}
b_{1}=\sum_{\alpha \in I} \sum_{\beta \in B_{\alpha}} p_{\alpha} p_{\beta}, \\
b_{2}=\sum_{\alpha \in I} \sum_{\alpha \neq \beta \in B_{\alpha}} \mathbb{E}\left[X_{\alpha} X_{\beta}\right],
\end{gathered}
$$

$\mathrm{e}$

$$
b_{3}=\sum_{\alpha \in I} \mathbb{E}\left|\mathbb{E}\left\{X_{\alpha}-p_{\alpha} \mid \sigma\left(X_{\beta}: \beta \notin B_{\alpha}\right)\right\}\right| .
$$

Então, vale o seguinte teorema 
Teorema 5.6 (Arratia, Goldstein e Gordon, 1989) Seja $W=\sum_{\alpha \in I} X_{\alpha}$ o número de ocorrências de eventos dependentes e seja $Z$ uma variável aleatória com distribuição de Poisson com $\mathbb{E} Z=\mathbb{E} W=\lambda<\infty$. Então

$$
\begin{aligned}
\|\mathcal{L}(W)-\mathcal{L}(Z)\| & \leq 2\left[\left(b_{1}+b_{2}\right) \frac{1-e^{-\lambda}}{\lambda}+b_{3}\left(1 \wedge 1.4 \lambda^{-1 / 2}\right)\right] \\
& \leq 2\left(b_{1}+b_{2}+b_{3}\right)
\end{aligned}
$$

e

$$
\left|I P\{W=0\}-e^{-\lambda}\right| \leq\left(b_{1}+b_{2}+b_{3}\right) \frac{1-e^{-\lambda}}{\lambda} .
$$

No entanto, é preciso apresentar expressões mais transparentes para as quantidades $b_{1}, b_{2}$ e $b_{3}$, assim como condições suficientes para garantir sua convergência a zero para o teorema resultar interessante. É este assunto o que consideraremos na próxima seção.

\subsection{Convergência a um processo de Poisson para processos mis- turadores}

O método de Chen-Stein para soma de variáveis aleatórias dependentes pode ser comparado ao método de Hirata, Saussol e Vaienti para o primeiro instante de ocorrência de um evento raro no seguinte sentido. O termo $b_{1}$ refere-se ao tamanho de uma vizinhança em torno do evento raro. O termo $b_{3}$ refere-se às correlações longas que devem ser pequenas se o processo perde memória suficientemente rápido. No entanto, o termo $b_{2}$ refere-se às correlações curtas. E esta quantidade pode não convergir para zero se o evento considerado é recorrente. Esta informação deve ser colocada na re-escalação do processo tal e como foi feita nos trabalhos de Galves e Schmitt (1997) e Abadi (2000) para lograr a convergência em para eventos não recorrentes e também para os recorrentes. Neste último caso, o tipo de processo que se terá, então, não é um processo de Poisson e sim, um processo marcado de Poisson. Devemos, portanto, considerar somente eventos que não sejam deste tipo.

Temos então, o seguinte teorema: 
Teorema 5.7 Seja $\left\{X_{t}\right\}_{t \in \mathbb{Z}}$ um processo estocástico $\phi$-misturador. Sejam $A \notin \mathcal{B}(2)$. Então o processo das sucessivas ocorrências do evento $A$ até o instante $t$, converge em distribuição para um processo de Poisson. Mais ainda

$$
\left|\mathbb{P}\left\{N_{\left[\frac{t}{P\{A\}}\right]}=k\right\}-\frac{e^{-t} t^{k}}{k !}\right| \leq C\left[\inf _{\Delta}[\Delta \mathbb{P}\{A\}+\phi(\Delta)]+n P\left\{A^{(n / 2)}\right\}\right] \sqrt{t}
$$

Prova. Para provar este teorema será suficiente obter majorações para $b_{1}, b_{2}$ e $b_{3}$ no método de Chen-Stein.

O conjunto de índices é $I=\{0,1, \ldots, t-1\}$. Posto que a medida é invariante temos que $p_{\alpha}=p_{\beta}=\mathbb{P}\{A\}$. Seja $\Delta>n$. Tomamos a vizinhança em cada instante $\alpha$ como sendo $B_{\alpha}=[\alpha-\Delta, \alpha+n-1+\Delta] \cap I$, para todo $\alpha$. Escrevemos também $B=B_{0}$. De esta maneira, temos que

$$
b_{1} \leq t(2 \Delta+n) \mathbb{P}\{A\}^{2} \leq 3 \Delta \mathbb{P}\{A\}^{2} t
$$

$$
\begin{aligned}
b_{2} \leq t \sum_{j \in B} \mathbb{E}\left[\mathbb{I}_{\left\{T^{-j} A\right\}} \mathbb{I}_{\{A\}}\right] & \leq t \sum_{0 \neq j=-\Delta}^{\Delta+n-1} \mathbb{P}\left\{T^{-j} A \cap A\right\} \\
& \leq t 2 \sum_{j=1}^{\Delta+n-1} \mathbb{P}\left\{T^{-j} A \cap A\right\}
\end{aligned}
$$

Posto que $A \notin \mathcal{B}(2)$ temos que para $j=1, \ldots, n / 2$,

$$
\mathbb{P}\left\{T^{-j} A \cap A\right\}=0
$$

Podemos controlar os termos com $j=n / 2+1, \ldots, n$, como no Lema. 3.8 


$$
\begin{aligned}
\sum_{j=n / 2+1}^{n} \mathbb{P}\left\{T^{-j} A \cap A\right\} & \leq \sum_{j=n / 2+1}^{n}(1+\phi(0)) \mathbb{P}\{A\} \mathbb{P}\left\{A^{(j)}\right\} \\
& \leq C \mathbb{P}\{A\} n P\left\{A^{(n / 2)}\right\}
\end{aligned}
$$

Similarmente, o resto dos termos da somatória são controlados como no Lema 3.8

$$
\sum_{j=n}^{\Delta+n-1} \mathbb{P}\left\{T^{-j} A \cap A\right\} \leq \sum_{j=n}^{\Delta+n-1}(1+\phi(0)) \mathbb{P}\{A\}^{2} \leq C \Delta \mathbb{P}\{A\}^{2}
$$

Pelo que

$$
b_{2} \leq C t \mathbb{P}\{A\}\left[\Delta \mathbb{P}\{A\}+n P\left\{A^{(n / 2)}\right\}\right] .
$$

Finalmente,

$$
b_{3}=t \mathbb{E}\left|\mathbb{E}\left\{\mathbb{I}_{\{A\}}-\mathbb{P}\{A\} \mid \sigma\left(X_{t}: t \notin B\right)\right\}\right|
$$

A esperança no lado direito da igualdade é

$$
\begin{aligned}
& \sum_{Y=y} \mathbb{P}\{Y=y\}\left|\mathbb{E}\left\{\mathbb{1}_{\{A\}}-\mathbb{P}\{A\} \mid Y=y\right\}\right| \\
= & \sum_{y} \mathbb{P}\{Y=y\}|\mathbb{P}\{A \mid Y=y\}-\mathbb{P}\{A\}| \\
= & \sum_{y}|\mathbb{P}\{A \cap Y=y\}-\mathbb{P}\{A\} \mathbb{P}\{Y=y\}|,
\end{aligned}
$$


onde $Y=\left\{X_{t}\right\}_{t \notin B}$. Na igualdade acima, podemos supor que $B^{c}$ possui uma quantidade finita de coordenadas e logo usar o clássico teorema de convergência de martingais para obter a igualdade para infinitas coordenadas. Entanto podemos escrever

$$
\{Y=y\}=\left\{Y_{e}=y_{e}\right\} \cap\left\{Y_{d}=y_{d}\right\}
$$

onde $Y_{e}=\left\{X_{t}\right\}_{t<-\Delta}$ e $Y_{d}=\left\{X_{t}\right\}_{t>\Delta+n-1}$ (e equivalentemente para $y_{e}$ e $y_{d}$ ), e usando a desigualdade triangular

$$
\begin{aligned}
& |\mathbb{P}\{A \cap Y=y\}-\mathbb{P}\{A\} \mathbb{P}\{Y=y\}| \\
\leq & \left|\mathbb{P}\{A \cap Y=y\}-\mathbb{P}\left\{A \cap Y_{e}=y_{e}\right\} \mathbb{P}\left\{Y_{d}=y_{d}\right\}\right| \\
+ & \left|\mathbb{P}\left\{A \cap Y_{e}=y_{e}\right\} \mathbb{P}\left\{Y_{d}=y_{d}\right\}-\mathbb{P}\{A\} \mathbb{P}\left\{Y_{e}=y_{e}\right\} \mathbb{P}\left\{Y_{d}=y_{d}\right\}\right| \\
+ & \left|\mathbb{P}\{A\} \mathbb{P}\left\{Y_{e}=y_{e}\right\} \mathbb{P}\left\{Y_{d}=y_{d}\right\}-\mathbb{P}\{A\} \mathbb{P}\{Y=y\}\right| .
\end{aligned}
$$

Agora, a propriedade de mistura nos dá a seguinte majoração

$$
\begin{aligned}
|\mathbb{P}\{A \cap Y=y\}-\mathbb{P}\{A\} \mathbb{P}\{Y=y\}| & \leq \phi(\Delta) \mathbb{P}\left\{A \cap Y_{e}=y_{e}\right\} \mathbb{P}\left\{Y_{d}=y_{d}\right\} \\
& +\phi(\Delta) \mathbb{P}\{A\} \mathbb{P}\left\{Y_{e}=y_{e}\right\} \mathbb{P}\left\{Y_{d}=y_{d}\right\} \\
& +\phi(2 \Delta+n) \mathbb{P}\{A\} \mathbb{P}\left\{Y_{e}=y_{e}\right\} \mathbb{P}\left\{Y_{d}=y_{d}\right\}
\end{aligned}
$$

Também pela propriedade de mistura

$$
\mathbb{P}\left\{A \cap Y_{e}=y_{e}\right\} \leq(1+\phi(\Delta)) \mathbb{P}\{A\} \mathbb{P}\left\{Y_{e}=y_{e}\right\}
$$

e da mesma maneira

$$
\mathbb{P}\left\{Y_{e}=y_{e}\right\} \mathbb{P}\left\{Y_{d}=y_{d}\right\} \leq \frac{\mathbb{P}\{Y=y\}}{1-\phi(2 \Delta+n)}
$$


Obtemos finalmente que

$$
\begin{aligned}
\sum_{y}|\mathbb{P}\{A \cap Y=y\}-\mathbb{P}\{A\} \mathbb{P}\{Y=y\}| & \leq \sum_{y} \frac{4 \phi(\Delta) \mathbb{P}\{A\}}{1-\phi(2 \Delta+n)} \mathbb{P}\{Y=y\} \\
& \leq C \phi(\Delta) \mathbb{P}\{A\}
\end{aligned}
$$

Assim

$$
b_{3} \leq C t \phi(\Delta) \mathbb{P}\{A\} .
$$

A soma das majorações para $b_{1}, b_{2}$ e $b_{3}$ e a mudança de variáveis $s=\mathbb{P}\{A\} t$, finaliza a prova do teorema.

Observação $1 \mathrm{O}$ uso no teorema de cilindros não pertencentes ao $\mathcal{B}_{n}(s)$ com o valor $s=2$, não é estritamente necessário. Qualquer outro valor de $s$ dará um resultado semelhante, salvo o valor da constante $C$ e da "porção de cilindro $\mathbb{I P}\left\{A^{(n / s)}\right\}$ " no terceiro termo da majoração do teorema.

Observação 2 A hipótese $A \in \mathcal{B}_{n}(s)$ para algum $s$ natural é necessária. Veremos isso no próximo exemplo.

Exemplo 5.8 Consideremos uma cadeia de Markov com dois estados $\{0,1\}$. Suponhamos que a matriz de transição da cadeia esta dada por

$$
Q=\left[\begin{array}{cc}
1-p & p \\
q & 1-q
\end{array}\right]
$$

Para todo $n$ natural sejam $A_{n}=\left\{X_{0}=1, X_{1}=0, X_{2}=1, X_{3}=0, \ldots\right\}$. Então para $j=$ $0, \ldots, n-1$,

$$
\mathbb{P}\left\{T^{-j} A \cap A\right\}=\left\{\begin{array}{lll}
0 & j & \text { impar } \\
\operatorname{IP}\{A\} Q(0,1)^{j / 2} Q(1,0)^{j / 2} & j & \text { par }
\end{array}\right.
$$


Portanto,

$$
b_{2} \geq C t \sum_{j=1}^{n / 2} \mathbb{P}\left\{T^{-j} A \cap A\right\} \geq C t \mathbb{P}\{A\} \sum_{j=1}^{n / 4} Q(0,1)^{j} Q(1,0)^{j} \geq C t \mathbb{P}\{A\}
$$

o que mostra, após o re-escalamento $s=t \mathbb{P}\{A\}$, que $b_{2}$ não converge para zero.

É importante mostrar que tipo de resultado para o instante de entrada deriva-se do Teorema 5.7 .

Temos o seguinte corolário

Corolário 5.9 Baixo as condições acima, para todo $t$ real, se satisfaz a seguinte desigualdade,

$$
\begin{aligned}
\left|\mathbb{P}\left\{\tau_{A}>\frac{t}{\mathbb{P}\{A\}}\right\}-e^{-t}\right| & \leq C\left(\inf _{\Delta}[\Delta \mathbb{P}\{A\}+\phi(\Delta)]+n P\left\{A^{(n / 2)}\right\}\right)(t \wedge 1) \\
& +C \mathbb{P}\{A\} e^{-t}
\end{aligned}
$$

Prova. Só é suficiente notar que, para $m \in \mathbb{I N}$

$$
\mathbb{P}\left\{N_{m}=0\right\}=\mathbb{P}\left\{\tau_{A}>m\right\}
$$

e aplicar a segunda parte do Teorema 5.6.

Para $t$ qualquer

$$
\mathbb{I P}\left\{\tau_{A}>t\right\}=\mathbb{P}\left\{\tau_{A}>[t]\right\}
$$

e 


$$
\left|e^{-[t] \boldsymbol{P}\{A\}}-e^{-t \boldsymbol{P}\{A\}}\right| \leq(t-[t]) \mathbb{P}\{A\} e^{-[t] P\{A\}} \leq C \mathbb{P}\{A\} e^{-t \boldsymbol{P}\{A\}}
$$

Isto e uma mudança de variáveis, conclui a prova do corolário.

Observação É importante comparar o erro que presenta o Corolário 5.9 para a aproximação exponencial do instante de entrada com o erro do Teorema 3.2. Mediante uma mudança de variáveis expressamos este último como

$$
\left|\mathbb{P}\left\{\tau_{A}>\frac{t}{\xi_{A} I P\{A\}}\right\}-e^{-t}\right| \leq C \Delta \mathbb{P}\{A\} t e^{-t}+C \Delta \mathbb{P}\{A\} e^{-t}
$$

Desta maneira, e para $A \notin \mathcal{B}(s)$, temos a seguinte situação.

Para $0 \leq t \leq 1 / \Delta$, o erro do método Chen-Sein é da ordem $\mathbb{P}\{A\}+n P\left\{A^{(n / s)}\right\} t$. No entanto, o erro do Teorema 3.2 é da ordem $\triangle \mathbb{P}\{A\}$.

Para $1 / \Delta \leq t \leq 1$, o erro do método Chen-Sein é da ordem $\Delta \mathbb{P}\{A\} t+n P\left\{A^{(n / s)}\right\} t$. No entanto, o erro do Teorema 3.2 ainda é da ordem $\triangle \mathbb{P}\{A\}$.

Para $t \geq 1$, o erro do método Chen-Sein é da ordem $\Delta \mathbb{P}\{A\}+n P\left\{A^{(n / s)}\right\}$, no entanto, o erro do Teorema 3.2 é da ordem $\triangle \mathbb{P}\{A\} t e^{-t}$.

Desta forma, para o instante de entrada, o Teorema 3.2 apressenta um resultado mais ajustado que o método de Chen-Stein para $t \geq 1$. Para $t<1$, dependerá da perda de correlação do sistema assim como das propriedades de recorrência do evento. 


\section{Referências Bibliográficas}

[1] M. Abadi (2000). Exponential approximation for hitting times in mixing processes. Relatório técnico IME-USP. Pode ser baixado de http://rene.ma.utexas.edu/mp_arc/c /00/00-211.ps.gz

[2] M. Abadi e A. Galves (2000). Inequalities for the occurrence times of rare events in mixing processes. The state of the art Markov Proc. Relat. Fields. Por aparecer. Pode ser baixado de $h t t p: / / w w w . m a . u t e x a s . e d u / m p \_a r c-b i n / m p a ? y n=00-262$.

[3] D. J. Aldous (1982). Markov chains with almost exponential hitting times. Stochastic Process. Appl. 13, 305-310.

[4] D. J. Aldous e M. Brown (1992). Inequalities for rare events in time-reversible Markov chains I. Stochastic Inequalities IMS Lecture Notes 22, 1-16.

[5] D. J. Aldous e M. Brown (1993). Inequalities for rare events in time-reversible Markov chains II. Stochastic Processes Appl. 44, 15-25.

[6] D. Arratia, M. Goldstein e Gordon (1990). Poisson approximation and the Chen-Stein method. With comments and a rejoinder by the authors. Stat. sci. 5, 403-434.

[7] A. Asselah e P. Dai Pra (1997a). Sharp estimates for the occurrence time of rare events for simple symmetric exclusion. Stochastic Processes Appl. 71, 259-273.

[8] A. Asselah e P. Dai Pra (1997b). Ocurrence of rare events in ergodic interacting spin systems. Ann. Inst. H. Poincaré 33 6, 727-751.

[9] R. Bellman e T. E. Harris (1951). Recurrence times for the Ehrenfest model. Pacific J. Math. 1, 179-193.

[10] P. Billingsley (1965). Ergodic theory and information. Wiley, New York.

[11] R. Bowen (1975). Equilibrium states and the ergodic theory of Anosov diffeomorphisms. Lecture Notes in Math. 470. Springer-Verlag, New York. 
[12] X. Bressaud, R. Fernandez e A. Galves (1999). Speed of $\bar{d}$-convergence for Markov approximations of chains with complete connections. A coupling approach. Stochastic Process. Appl. 83. 1, 127-138.

[13] X. Bressaud e R. Zweimüller (2000). Non exponential law of entrance times in asymptotically rare for intermittent maps with infinite invariante measure. Relatório técnico, IML-Luminy-Marseille.

[14] M. Cassandro, A. Galves, E. Olivieri e M. E. Vares (1984). Metastable behavior of stochastic dynamics: a pathwise approach. J. Stat. Phys. 35, 603-633.

[15] L. Chen (1975). Poisson approximation for dependent trials. Ann. Probab. 3, 534-345.

[16] R. Cogburn (1985). On the distribution of first passage and return times for small sets. Ann. Probab. 13, 1219-1223.

[17] Z. Coelho e P. Collet (1994). Asymptotic limit law for the close approach of two trajectories in expanding maps of the circle. Probab. Theory. Relat. Fields.99, 237-250.

[18] P. Collet (2000). Statistics of close return for non uniformly hyperbolic systems. Relatório técnico CPT - CNRS, Ecole Polytéchnique.

[19] P. Collet e A. Galves (1993). Statistics of close visits to the indifferent fixed point of an interval map. J. Stat.Phys. 72, 459-478.

[20] P. Collet e A. Galves (1995) Asymptotic distribution of entrance times for expanding maps of the interval. Dynamical Systems and Applications, WSSIAA 4, 139-152.

[21] P. Collet, A. Galves e B. Schmitt (1992). Unpredictability of the occurrence time of a long laminar period in a model of temporal intermittency. Ann. Inst. H. Poincaré, Section A, 57 3, 319-331.

[22] Collet, A. Galves e B. Schmitt (1999). Repetition times for gibbsian sources. Nonlinearity vol. $12,1225-1237$.

[23] I. Cornfeld, S. Fomin e Y. Sinai (1982). Ergodic theory, vol 245. Springer-Verlag, New York.

[24] W. Döeblin (1940)..Remarques sur la théorie métrique des fractions continues. Compositio Math. 7, 353-371.

[25] P. Doukhan (1995). Mixing. Properties and examples. Lecture Notes in Statistics 85. Springer-Verlag.

[26] W. Feller (1976). Introdução à teoria das probabilidades e suas aplicações E. Blucher São Paulo. 
[27] P. A. Ferrari, A. Galves (1997). Acoplamento e Processos Estocásticos. 21 Coloquio Brasileiro de Matematica IMPA.

[28] P. A. Ferrari, A. Galves e C. Landim (1994). Exponential waiting time for a big gap in a one dimensional zero range process. Ann. Probab. 22 1, 284-288.

[29] P. A. Ferrari, A. Galves e T. Liggett (1995). Exponential waiting time for filling a large interval in the symmetric simple exclusion process. Ann. Inst. H. Poincaré 31 1, 155-175.

[30] A. Galves e B. Schmitt (1990). Ocurrence times of rare events for mixing dynamical systems. Ann. Inst. H. Poincaré 52 3, 267-281.

[31] A. Galves e B. Schmitt (1997). Inequalities for hitting times in mixing dynamical systems. Random Comput. Dyn. 5, 337-348.

[32] N. Haydn (1999). The distribution of the first return time for rational maps. J. Stat. Phys. 94 5-6, 1027-1036.

[33] N. Haydn e S. Vaienti (2000). The distribution of the first return time for rational maps. Relatório técnico, CPT-CNRS, Marseille.

[34] M. Hirata (1993). Poisson law for Axiom A diffeomorphism. Ergod. Th. Dyn. Sys. 13, 533-556.

[35] M. Hirata, B. Saussol e S. Vaienti (1999). Statistics of return times: a general framework and new applications. Comm. Math. Phys. 206, 33-55.

[36] T. E. Harris (1953). First passage and recurrence distributions. Trans. Amer. Math. Soc. 73, 471-486.

[37] I. A. Ibragimov (1962). Some limit theorems for stationary processes. Theory Prob. Appl. 7, 349-382.

[38] M. Kac (1947). On the notion of recurrence in discrete stochastic processes. Bull. Amer. Math. Soc. 53, 1002-1010.

[39] J. Keilson (1979). Markov Chain Models. Rarity and Exponentiality. Springer-Verlag.

[40] C. Kipnis e C. Newman (1985). The metastable behavior of infrequently observed, weakly random, one-dimensional diffusion processes. SIAM J. Appl. Math. 45, 972.

[41] I. Kontoyiannis (1998). Asymptotic Recurrence and waiting times for stationary processes. J. Theoret. Probab. 11, no. 3, 795-811.

[42] D. V. Korolyuk e D. S. Sil'vestrov (1984). Entry times into asymptotically receding domains for ergodic Markov chains. Theory Prob. Appl. 19, 432-442. 
[43] J. L. Lebowitz e R. H. Schonmann (1987). On the asymptotics of occurrence times of rare events for stochastic spin systems. J. Stat. Phys. 48, 727-751.

[44] E. Olivieri e E. Scoppola (1995). Markov chains with exponentially small transition probabilities: first exit problem from a general domain. I. The reversible case. J. Stat. Phys. 79, 613-647.

[45] E. Olivieri e E. Scoppola (1996) Markov chains with exponentially small transition probabilities: first exit problem from a general domain. II. The general case. J. Statist. Phys. 84 , no. 5-6, 987-1041.

[46] F. Paccaut (1999). Statistics of return times for weighted maps of the interval. Relatório técnico Laboratorie de Topologie, Université de Bourgogne.

[47] W. Philipp e W. Stout (1975). Almost sure invariance principles for partial sums of weakly dependant random variables. Memoirs of Amer. Math. Soc. 2, 161.

[48] B. Pitskel (1991). Poisson law for Markov chains. Ergod. Th. Dyn. Sys. 11, 501-513.

[49] B. Saussol (1998). Tese de doutorado. Centre de Physique Téorique CNRS. MarseilleLuminy. Université de Toulon et du Var.

[50] B. Schmitt (2000). Poincaré and isoperimetric inequalities for $g$-measures. Spectral gap. Concentration phenomenon. Relatório técnico. Workshop on Dynamics and Randomness. Centro de Modelamiento Matemático, Universidad de Chile, UMR-CNRS.

[51] P. Shields (1996). The ergodic theory of discrete sample paths. Graduate Studies in Mathematics 13 A.M.S.

[52] A. Simonis (1998). Filling the hypercube in the supercritical contact process in equilibrium. Markov Process. Rel. Fields 41, 113-130.

[53] H. Weyl (1916). Úber der Gleichverteilung von Zahlen mod. 1. Math. Ann. 77 313-352. 